Estimação e diagnóstico na distribuição exponencial por partes em análise de sobrevivência com fração de cura 


\title{
Estimação e diagnóstico na distribuição exponencial por partes em análise de sobrevivência com fração de cura
}

\author{
Alessandra Cristiane Sibim
}

Orientador: Prof. Dr. Vicente Garibay Cancho

Dissertação apresentada ao Instituto de Ciências Matemáticas e de Computação - ICMC-USP, como parte dos requisitos para obtenção do título de Mestre em Ciências - Ciências de Computação e Matemática Computacional. VERSÃO REVISADA.

USP - São Carlos

Maio/2011 
Ficha catalográfica elaborada pela Biblioteca Prof. Achille Bassi e Seção Técnica de Informática, ICMC/USP, com os dados fornecidos pelo(a) autor(a)

Sibim, Alessandra Cristiane

Estimação e diagnóstico na distribuição exponencial por partes em análise de sobrevivência com fração de cura / Alessandra Cristiane Sibim; orientador Vicente Garibay Cancho -- São Carlos, 2011. $63 \mathrm{p}$.

Dissertação (Mestrado - Programa de Pós-Graduação em Ciências de Computação e Matemática Computacional) -Instituto de Ciências Matemáticas e de Computação, Universidade de São Paulo, 2011.

1. Análise de sobrevivência. 2. Inferência bayesiana. 3. Divergência de Kullback-Leibler. 4. Métodos MCMC. I. Cancho, Vicente Garibay, orient. II. Título. 
Aos meus pais e irmãos. 


\section{Agradecimentos}

Primeiramente agradeço a Deus, que me dá saúde e força para superar os obstáculos todos os dias.

Aos meus pais que sempre me ajudam, apoiam e me incentivam na árdua tarefa de estudar e a quem sempre estarei em dívida.

Ao meu orientador, Vicente Garibay Cancho, quero agradecer por ter acreditado em mim e por estar presente em cada etapa deste trabalho. Agradeço ainda pela paciência, incentivo na elaboração e condução do trabalho, portanto tem minha eterna gratidão e admiração.

Aos professores Francisco Louzada Neto e Mário de Castro, membros da banca do exame de qualificação, pelas sugestões feitas.

Aos professores e funcionários da biblioteca Prof. Achille Bassi e da pós-graduação do ICMC pelo excelente convívio.

Aos professores Carlos Aparecido dos Santos e Jacinta Ludovico Zamboti pelos primeiros ensinamentos estatísticos e pelo incentivo que me levaram a fazer a pósgraduação.

Em especial ao Wesley pelo apoio e incentivo na realização deste sonho, a Maria Inês pela grande ajuda que viabilizou a obtenção de resultados e a elaboração deste texto, a Conceição pelas doces palavras em um momento difícil.

A todos os meus amigos do curso de pós graduação Adriana, Aline, Gilberto, Marcia e William pelos momentos compartilhados no decorrer destes dois anos. 
Aos demais amigos que, independentemente da distância, acompanharam-me nesta jornada. Não citarei nomes, pois a lista seria muito extensa para ser colocada aqui. Além disso, não correrei o risco de acabar esquecendo de alguém.

A todas as pessoas que não foram nominalmente mencionadas, mas que de alguma forma contribuíram para viabilizar este trabalho.

Finalmente, agradeço à Fundação de Amparo à Pesquisa do Estado de São Paulo (FAPESP) pelo suporte financeiro concedido para a realização deste trabalho. 
"Feliz aquele que transfere o que sabe e aprende o que ensina".

"O saber se aprende com os mestres. A sabedoria, só com o corriqueiro da vida". 


\section{Resumo}

O principal objetivo deste trabalho é desenvolver procedimentos inferências em uma perspectiva bayesiana para modelos de sobrevivência com (ou sem) fração de cura baseada na distribuição exponencial por partes. A metodologia bayesiana é baseada em métodos de Monte Carlo via Cadeias de Markov (MCMC). Para detectar observações influentes nos modelos considerados foi usado o método bayesiano de análise de influência caso a caso (Cho et al., 2009), baseados na divergência de Kullback-Leibler. Além disso, propomos o modelo destrutivo binomial negativo com fração de cura. O modelo proposto é mais geral que os modelos de sobrevivência com fração de cura, já que permitem estimar a probabilidade do número de causas que não foram eliminadas por um tratamento inicial. Palavras-chave: Inferência Bayesiana, Medidas de Diagnóstico Bayesiano, Divergência de Kullback-Leibler, Métodos MCMC, Análise de Sobrevivência. 


\section{Abstract}

The main objective is to develop procedures inferences in a bayesian perspective for survival models with (or without) the cure rate based on piecewise exponential distribution. The methodology is based on bayesian methods for Markov Chain Monte Carlo (MCMC). To detect influential observations in the models considering bayesian case deletion influence diagnostics based on the Kullback-Leibler divergence (Cho et al., 2009). Furthermore, we propose the negative binomial model destructive cure rate. The proposed model is more general than the survival models with cure rate, since the probability to estimate the number of cases which were not eliminated by an initial treatment.

Keywords: Bayesian Inference, Measures of Diagnostic Bayesian, Kullback-Leibler Divergence, MCMC Methods, Survival Analysis 


\section{Lista de Figuras}

2.1 (a) Função de sobrevivência e (b) função de taxa de falha para o MEP. . .

2.2 Gráfico de índices de $K\left(P, P_{(-i)}\right)$ para os dados simulados. . . . . . . . . . 19

2.3 Gráfico TTT plot para dados de infecção renal. . . . . . . . . . . . . . . 20

2.4 Gráfico de índices de $K\left(P, P_{-i}\right)$ para os dados de infecção renal. . . . . . . . 22

2.5 Estimativas da função de sobrevivência por sexo: (a) sexo masculino e (b)

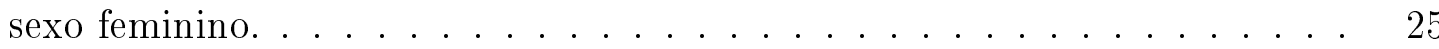

3.1 Gráfico de índices de $K\left(P, P_{(-i)}\right)$ para os dados simulados com fração de cura. 35

3.2 Estimativa de Kaplan-Meier da função de sobrevivência por categoria nódulo (1 a 4 , de cima para baixo). . . . . . . . . . . . . . . . 36

3.3 Gráfico de índices de $K\left(P, P_{-i}\right)$ para dados de melanoma. . . . . . . . . . . 38

3.4 Proporção de curados por categoria nódulo (1 a 4, de cima para baixo). . . 42

4.1 Gráfico de índices de $K\left(P, P_{-i}\right)$ para dados de melanoma considerando o modelo destrutivo. . . . . . . . . . . . . . . . . . . . 51

4.2 Proporção de curados por categoria nódulo (1 a 4, de cima para baixo). . . 55 


\section{Lista de Tabelas}

2.1 Médias e desvios padrão a posteriori para os parâmetros do modelo dos dados simulados.

2.2 Medidas de diagnósticos para os dados simulados. . . . . . . . . . . . . . 18

2.3 Critérios de seleção de modelos considerando os dados de infecção renal. . . 21

2.4 Resumos a posteriori para os tempos. . . . . . . . . . . . . . . . . 21

2.5 Identificação dos casos influentes para dados de infecção renal. . . . . . . . . 22

2.6 Estimativas, $V R$ (em \%) e a correspondente HPD (95\%) ajustados para o conjunto de dados de infecção renal. . . . . . . . . . . . . . . . . . . . . . 23

2.7 Resumo a posteriori para o modelo final. . . . . . . . . . . . . . . . . . . . 24

3.1 Médias e desvios padrão a posteriori para os parâmetros do modelo dos dados simulados com fração de cura. . . . . . . . . . . . . . . . . . . . . . 33

3.2 Critérios de seleção de modelos. . . . . . . . . . . . . . . . . . . . 33

3.3 Medidas de diagnósticos para os dados simulados com fração de cura . . . . 34

3.4 Critérios de seleção de modelos de acordo com cada partição. . . . . . . . . 37

3.5 Resumo a posteriori dos parâmetros do modelo. . . . . . . . . . . . . . . 38

3.6 Identificação dos casos influentes para dados de melanoma. . . . . . . . . . 39 
3.7 Estimativas, $V R($ em \%) e a correspondente HPD (90\%) ajustados para o conjunto de dados de melanoma. . . . . . . . . . . . . . . . 40

3.8 Resumo dos parâmetros a posteriori para o modelo final. . . . . . . . . . . 41

3.9 Resumo a posteriori para a fração de cura estratificada por categoria do nódulo. . . . . . . . . . . . . . . . . . .

3.10 Resumo a posteriori para a fração de cura $\left(p_{0}\right)$ estratificada por categoria do nódulo e por idade. . . . . . . . . . . . . . . . . . . . . . . . . 42

4.1 Critérios de seleção para o modelo destrutivo. . . . . . . . . . . . . . . 50

4.2 Estimativas a posteriori para o modelo MEP destrutivo. . . . . . . . . . 50

4.3 Identificação dos casos influentes para dados de melanoma. . . . . . . . . . 52

4.4 Estimativas, VR (em \%) e a correspondente HPD (90\%) ajustados para o conjunto de dados de melanoma. . . . . . . . . . . . . . . . . . 53

4.5 Resumo dos parâmetros a posteriori para o modelo final. . . . . . . . . . . 54

4.6 Resumo a posteriori para a probabilidade da presença de causas competindo $(p)$ estratificada por categoria do nódulo. . . . . . . . . . . . . . 54

4.7 Resumo a posteriori para a fração de cura $\left(p_{0}\right)$ estratificada por categoria do nódulo e por idade. . . . . . . . . . . . . . . . . . . . 55 


\section{Sumário}

1 Introdução .......................... 1

1.1 Critérios de comparação de modelos . . . . . . . . . . . . . . . 5

1.2 Diagnóstico . . . . . . . . . . . . . . . . . . . . . 6

1.2.1 Análise de influência caso a caso . . . . . . . . . . . . . . . . 7

1.3 Organização dos capítulos . . . . . . . . . . . . . . . . . . . . . . 9

2 Modelo Exponencial por Partes ................. 11

2.1 Formulação do modelo . . . . . . . . . . . . . . . . . . . . . . 11

2.2 Modelo de regressão semiparamétrico (MRSP) . . . . . . . . . . . . . . 13

2.3 Inferência bayesiana . . . . . . . . . . . . . . . . . . . . . . . . 14

2.4 Aplicação . . . . . . . . . . . . . . . . . . . . . . 16

2.4.1 Dados simulados . . . . . . . . . . . . . . . . 16

2.4.2 Dados de infecção renal . . . . . . . . . . . . . . . . . . . . . . 19

2.5 Conclusões . . . . . . . . . . . . . . . . . . . . . . . 25

3 Modelo de sobrevivência com fração de cura . . . . . . . . . . . 27

3.1 Formulação do modelo . . . . . . . . . . . . . . . . . . . . . . . . . . 27

3.2 Função de Verossimilhança . . . . . . . . . . . . . . . . . . . . . 30 
3.3 Inferência bayesiana . . . . . . . . . . . . . . . . . . . . . . 31

3.4 Aplicação . . . . . . . . . . . . . . . . . . . . . . . 32

3.4.1 Dados simulados . . . . . . . . . . . . . . . . . . . 32

3.4 .2 Dados de melanoma . . . . . . . . . . . . . . . . . 36

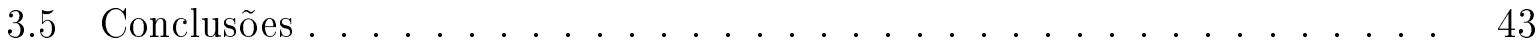

4 Um modelo destrutivo com fração de cura . . . . . . . . . . . 45

4.1 Formulação do modelo . . . . . . . . . . . . . . . . . . . . . . . . . 46

4.2 Inferência bayesiana . . . . . . . . . . . . . . . . . . . . . 47

4.3 Aplicação . . . . . . . . . . . . . . . . . . . . . . 49

4.4 Conclusões . . . . . . . . . . . . . . . . . . . . . . 56

5 Considerações Finais e Propostas Futuras $\ldots \ldots \ldots$

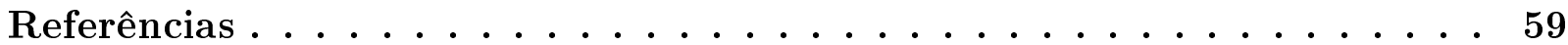




\section{Capítulo 1}

\section{Introdução}

Análise de Sobrevivência é uma das áreas da Estatística que mais cresceu nos últimos 20 anos. Este crescimento se justifica pelo desenvolvimento e aprimoramento de técnicas estatísticas combinados com avanços computacionais (Colosimo \& Giolo, 2006).

Análise de sobrevivência é o termo utilizado para designar a análise estatística de dados quando a variável em estudo representa o tempo a partir um instante inicial bem definido até à ocorrência de determinado evento de interesse denominado de falha.

É comum em análise de sobrevivência a presença de observações parciais ou incompletas, ou seja, observações que não apresentam o evento de interesse. Essas observações são denominadas observações censuradas e podem ocorrer por uma variedade de razões:

(i) A perda do acompanhamento do paciente no decorrer do estudo;

(ii) A não ocorrência do evento de interesse até o término do experimento;

(iii) O término do experimento antes da ocorrência do evento de interesse.

Mesmo censurados, todos os resultados provenientes de um estudo de sobrevivência devem ser usados na análise estatística, pois sua omissão pode acarretar conclusões viciadas e mesmo sendo incompletas, as observações censuradas fornecem informação sobre o tempo de vida dos indivíduos em estudo. As censuras são classificadas em censura do tipo I, 
censura do tipo II e censura do tipo aleatório. Mais detalhes podem ser encontrados em Colosimo \& Giolo (2006).

Ao se analisar dados de sobrevivência percebemos que muitas vezes há grande número de indivíduos censurados à direita. Empiricamente, esta característica é observada na estimativa da função de sobrevivência de Kaplan-Meier, que apresenta uma cauda direita em um nível aproximadamente constante e estritamente maior que 0 por um período considerável. Fato este que pode ser uma indicação de que em parte dos indivíduos em estudo o evento de interesse nunca ocorra, ou seja, uma fração da população apresenta imunidade. Isso ocorre, por exemplo em muitos estudos clínicos, especialmente em estudos de câncer, nos quais há uma fração significativa de pacientes que respondem favoravelmente ao tratamento e apresentam uma proporção de indivíduos sobreviventes ou curados, mesmo após um longo período de acompanhamento. Para tais dados de sobrevivência, uma proporção de indivíduos da população é suscetível ao evento de interesse e outros não o são. Nesta situação os modelos de sobrevivência usuais, que assumem que a função de sobrevivência converge para 0 quando o tempo de sobrevivência é suficientemente grande (função de sobrevivência própria), podem não ser adequados.

Uma abordagem bastante popular para modelar dados de sobrevivência com fração de cura (também chamados de modelos de sobrevivência de longa duração) é o modelo de mistura proposto por Boag (1949), posteriormente desenvolvido por Berkson \& Gage (1952), e que foi amplamente estudado por vários autores (vide, Maller \& Zhou, 1996).

A literatura sobre modelos de sobrevivência com fração de cura é extensa e está em rápido desenvolvimento. Podemos destacar como referências fundamentais os livros de Maller \& Zhou (1996) e Ibrahim et al. (2001), como também o artigo de Tsodikov et al. (2003) e o artigo de Cooner et al. (2007). Fora do contexto das aplicações a dados de estudos clínicos, Yamaguchi (1992) considera o modelo de mistura em estudos de desemprego, ao passo que Hoggart \& Griffin (2001) desenvolveram um modelo para o tempo até que um indivíduo deixe de ser cliente de um banco. Zaider et al. (2001) estudam modelos de longa duração adotando como base conceitos de processos estocásticos. Chen \& Ibrahim 
(2001) discutem estimação por máxima verossimilhança em um modelo semiparamétrico. O modelo bayesiano de Chi \& Ibrahim (2007) estabelece uma flexibilidade na fração de cura, podendo esta ser nula ou positiva. Kim et al. (2007) propuseram um modelo semiparamétrico dinâmico bayesiano. Mizoi \& Bolfarine (2007) (vide ainda Mizoi, 2004) estenderam o modelo de tempo de promoção considerando situações em que há erros de medição nas covariáveis. Tournoud \& Ecochard (2007) tratam de um modelo com fração de cura em situações em que a exposição a um fator de risco ocorre em diversas ocasiões. Paes (2007) e Fonseca (2009) estudam modelos de sobrevivência com fração de cura com omissão nas covariáveis. Sen \& Tan (2008) discutem estimação não-paramétrica da fração de cura na presença de censura intervalar. Lopes (2008) apresenta dois modelos com fração de cura considerando a introdução de efeitos aleatórios, um baseado no modelo de mistura e outro no modelo de tempo de promoção. Rodrigues et al. (2009a) propõem um modelo de sobrevivência com fração de cura unificado. Rodrigues et al. (2009b) discutem o uso da distribuição Conway-Maxwell Poisson na estimação da proporção de curados. Ortega et al. (2009) apresentam um modelo de regressão log-gama generalizado reparametrizado para incluir indivíduos que apresentam fração de cura, estimando assim, os efeitos das covariáveis sobre os tempos e realizando uma análise de sensibilidade e de resíduos sob uma abordagem clássica. Cancho et al. (2009) realizam um estudo de diagnóstico sob a perspectiva clássica para um modelo de regressão log-Weibull exponenciada considerando a presença de indivíduos curados. de Castro et al. (2010) desenvolveram um aplicativo para estimação dos parâmetros de modelos de sobrevivência com fração de cura por meio de pacote gamlss em $R$ (R Development Core Team, 2010). Cancho et al. (2011) consideram um modelo de sobrevivência com fração de cura, baseada na distribuição binomial negativa, abrangendo como um caso especial o modelo tempo de promoção.

Rodrigues et al. (2010b) propuseram modelo destrutivo com taxa de cura, considerando a distribuição Poisson ponderada, para modelar o número inicial de causas ou riscos relacionadas à ocorrência de um particular evento de interesse. Em Rodrigues et al. (2010a) é considerada uma abordagem bayesiana para o modelo destrutivo Poisson ponderado. 
Muitas famílias de distribuição foram consideradas para os tempos de vida. Neste trabalho consideramos a distribuição exponencial por partes por ser um modelo bastante flexível.

O modelo exponencial por partes (MEP) é amplamente utilizado em análise de sobrevivência, para modelar dados onde o interesse é o tempo até a ocorrência de um determinado evento, podendo ser o tempo até a morte do paciente, até a cura ou ainda reincidência de uma doença, o que comumente ocorre em estudos clínicos sobre leucemia (Breslow, 1974), transplante de coração (Aitkin et al., 1983), epidemia de AIDS (Brookmeyer \& Goedert, 1989), mortalidade hospitalar (Clark \& Ryan, 2002), entre outros. São vários os trabalhos existentes na literatura que discutem a aplicação do MEP, Friedman (1982) considerou-o para modelar a função de risco basal do modelo de Cox, apresentando condições para a existência e distribuição assintótica dos estimadores de máxima verossimilhança (EMV) para os coeficientes da regressão e taxas de falha. Kim \& Proschan (1991) fazem um estudo comparativo entre um estimador para o MEP e o estimador de Kaplan-Meier discutindo vantagens e desvantagens de ambos considerando dados sem covariáveis. Gamerman (1994) em uma abordagem bayesiana dinâmica utiliza o MEP não paramétrico considerando uma relação estocástica entre os sucessivos intervalos do tempo, fazendo uma comparação entre as estimativas bayesianas e clássicas. Barbosa et al. (1996) descrevem o uso do modelo exponencial por partes em dados de tempos de vida acelerados utilizando a abordagem de modelos lineares generalizados. Reineke et al. (1999) comparam o desempenho de um estimador exponencial por partes com o estimador de Kaplan-Meier considerando dados censurados. Ibrahim et al. (2001) utilizam o método de máxima verossimilhança para o MEP considerando modelos de sobrevivência com fração de cura com dados faltantes, Chen et al. (2002) considerando modelos de sobrevivência com fração de cura para o estudo de câncer com dados clínicos, empregam o MEP, Yin (2005) propôs um modelo de sobrevivência com fração de cura baseado na transformação de Box-Cox considerando o modelo exponencial por partes, unificando o modelo de mistura padrão (Berkson \& Gage, 1952) e o modelo de tempo de promoção (Yakovlev \& Tsodikov, 1996). Schmidli et al. (2007) consideram o modelo em uma abordagem bayesiana com 
priori informativas em um estudo de tratamento clínico. Chen et al. (2008) utilizam um modelo de regressão logística para o estudo de caso-controle de reincidência de câncer de mama e desenvolvem uma abordagem geral baseado na pseudo-verossimilhança para acomodar os dados com covariável faltante considerando o MEP.

\subsection{Critérios de comparação de modelos}

Uma questão importante consiste na avaliação e escolha do modelo que melhor represente a situação em estudo. Neste trabalho utilizamos dois critérios de seleção de modelos, os quais especificamente, são usados na metodologia bayesiana em que as amostras das distribuições a posteriori para os parâmetros do modelo são obtidos usando métodos MCMC. A seguir descrevemos cada um deles.

A densidade preditiva condicional ordenada (CPO) é uma ferramenta de avaliação do modelo muito útil e extensamente usada na literatura estatística sob vários contextos (Ibrahim et al., 2001). Seja $\mathcal{D}$ os dados completos e $\mathcal{D}_{(-i)}$ os dados com a $i$-ésima observação excluída. Denotamos a densidade a posteriori de $\gamma$ dado $\mathcal{D}_{(-i)}$ por $\pi\left(\gamma \mid \mathcal{D}_{(-i)}\right)$, $i=1, \ldots, n$ e $\gamma$ é um vetor dos parâmetros. Assim podemos escrever a $C P O_{i}$ para a $i$-ésima observação como

$$
C P O_{i}=\int_{\Theta} g\left(t_{i} \mid \boldsymbol{\gamma}\right) \pi\left(\boldsymbol{\gamma} \mid \mathcal{D}_{(-i)}\right) d \boldsymbol{\gamma}=\left\{\int_{\Theta} \frac{\pi(\boldsymbol{\gamma} \mid \mathcal{D})}{g\left(t_{i} \mid \boldsymbol{\gamma}\right)} d \boldsymbol{\gamma}\right\}^{-1}
$$

em que $g\left(t_{i} \mid \gamma\right)$ é a função densidade de probabilidade. Para valores altos de $C P O_{i}$ temos um melhor ajuste do modelo. Uma estimativa de Monte Carlo para $C P O_{i}$ considerando uma amostra de tamanho $Q$ da distribuição a posteriori $\pi(\boldsymbol{\gamma} \mid \mathcal{D}),($ Chen et al., 2000) é dada por

$$
\widehat{C P O_{i}}=\left\{\frac{1}{Q} \sum_{q=1}^{Q} \frac{1}{g\left(t_{i} \mid \gamma_{q}\right)}\right\}^{-1}
$$

Como em Cancho et al. (2010) utilizamos a estatística $B=\sum_{i=1}^{n} \log \left(\widehat{C P O}_{i}\right)$ na seleção dos modelos, maiores valores de $B$ indicam o melhor modelo. 
O outro critério utilizado neste trabalho é o "Deviance Information Criterion" (DIC) (Spiegelhalter et al., 2002) o qual é baseado na média a posteriori da deviance, que pode ser aproximada por

$$
\bar{D}=\frac{1}{Q} \sum_{q=1}^{Q} D\left(\gamma_{q}\right) \quad \text { com } \quad D(\gamma)=-2 \sum_{i=1}^{n} \log \left[g\left(t_{i} \mid \gamma_{q}\right)\right]
$$

O critério DIC também pode ser aproximado considerando amostras MCMC, por $\widehat{D I C}=2 \bar{D}-\hat{D}$, no qual

$$
\hat{D}=D\left(\frac{1}{Q} \sum_{q=1}^{Q} \gamma_{q}\right)
$$

Menores valores de $D I C$ determinam os melhores modelos.

\subsection{Diagnóstico}

Uma maneira de avaliar as suposições feitas sobre o modelo e de detectar pontos influentes pode ser efetuada pela análise de diagnóstico, a qual iniciou-se com a análise de resíduos para detectar a presença de pontos extremos e avaliar a adequação da distribuição proposta para a variável resposta. Uma referência importante neste assunto é o artigo de Cox \& Snell (1968) que apresenta uma forma bastante geral de definir resíduos, usada até os dias atuais (Paula, 2004). Uma das propostas mais inovadoras nesta área foi apresentada por Cook (1986), que propôs avaliar a influência conjunta das observações sob pequenas perturbações no modelo, ao invés da avaliação pela retirada individual ou conjunta de pontos. Se essas perturbações causam efeitos desproporcionais, pode ser indício de que o modelo está mal ajustado ou que podem existir afastamentos sérios das suposições feitas para o mesmo (Carrasco, 2007). Embora a metodologia proposta por Cook (1986) venha sendo aplicada com sucesso em diferentes áreas da estatística aplicada, observamos que dependendo da complexidade do modelo a aplicação da metodologia envolve extensas manipulações algébricas e em alguns casos um duro trabalho computacional. Vários autores têm aplicado as técnicas de influência local, Ortega et al. (2003) apresentam uma aplicação 
a modelos de regressão log-gama generalizadas com observações censuradas. Labra et al. (2005) realizam um estudo de influência local em um modelo com erros de medição $t$ de Student com intercepto nulo. Ortega et al. (2006) aplicaram um estudo de influência local para o modelo de regressão logística.

Peng \& Dey (1995) apresentam duas distintas abordagens bayesianas para detectar observações influentes no ajuste de modelos de regressão, uma é baseada na distribuição a posteriori e a segunda baseada na distribuição preditiva. Quatro medidas específicas são propostas, entre elas, destacamos a divergência Kullback-Leibler (K-L). Recentemente, Cho et al. (2009), propuseram um método bayesiano de análise de influência caso a caso para dados de sobrevivência, baseado na divergência Kullback-Leibler, no qual desenvolveram medidas de diagnóstico para avaliar a influência de um caso nas distribuições a posteriori conjuntas e marginais fundamentadas na divergência Kullback-Leibler.

\subsubsection{Análise de influência caso a caso}

Uma maneira comum de avaliar a influência de uma observação no ajuste de um modelo é por meio da deleção de casos (Cook \& Weisberg, 1982).

Suponha que $K\left(P, P_{(-i)}\right)$ denota a divergência K-L entre $P$ e $P_{(-i)}$, em que $P$ denota a distribuição a posteriori de $\gamma$ para os dados completos e $P_{(-i)}$ é a distribuição a posteriori de $\gamma$ sem o $i$-ésimo caso. Especificamente,

$$
K\left(P, P_{(-i)}\right)=\int \pi(\boldsymbol{\gamma} \mid \mathcal{D}) \log \left\{\frac{\pi(\boldsymbol{\gamma} \mid \mathcal{D})}{\pi\left(\boldsymbol{\gamma} \mid \mathcal{D}_{(-i)}\right)}\right\} d \boldsymbol{\gamma}
$$

$K\left(P, P_{(-i)}\right)$ mede o efeito de omitir o $i$-ésimo caso dos dados completos na distribuição a posteriori de $\gamma$. Note que $K\left(P, P_{(-i)}\right) \neq K\left(P_{(-i)}, P\right)$ em geral. Após alguma álgebra, podemos demonstrar uma expressão simplificada para $K\left(P, P_{(-i)}\right)$ dada por,

$$
K\left(P, P_{(-i)}\right)=\log E_{\boldsymbol{\gamma}}\left[\frac{L\left(\boldsymbol{\gamma} \mid \mathcal{D}_{(-i)}\right)}{L(\boldsymbol{\gamma} \mid \mathcal{D})} \mid \mathcal{D}\right]+E_{\boldsymbol{\gamma}}\left[\log \left\{\frac{L(\boldsymbol{\gamma} \mid \mathcal{D})}{L\left(\boldsymbol{\gamma} \mid \mathcal{D}_{(-i)}\right)}\right\} \mid \mathcal{D}\right]
$$

em que, $E[\cdot \mid \mathcal{D}]$ representa a média a posteriori de $\gamma, L(\gamma \mid \mathcal{D})=\prod_{k=1}^{n} f\left(t_{k} \mid \gamma\right)$ a função 
verossimilhança para os dados completos e $L\left(\gamma \mid \mathcal{D}_{(-i)}\right)=\prod_{k=1, k \neq i}^{n} f\left(t_{k} \mid \gamma\right)$ a função verossimilhança sem a $i$-ésima observação. A equação (1.4) pode ser reescrita como

$$
\begin{aligned}
K\left(P, P_{(-i)}\right) & =\log E_{\boldsymbol{\gamma}}\left[\left\{g\left(t_{i} \mid \boldsymbol{\gamma}\right)\right\}^{-1} \mid \mathcal{D}\right]+E_{\boldsymbol{\gamma}}\left[\log \left\{g\left(t_{i} \mid \boldsymbol{\gamma}\right)\right\} \mid \mathcal{D}\right] \\
& =-\log \left(C P O_{i}\right)+E_{\gamma}\left[\log \left\{g\left(t_{i} \mid \boldsymbol{\gamma}\right)\right\} \mid \mathcal{D}\right]
\end{aligned}
$$

Da Equação (1.5) uma estimativa de Monte Carlo para $K\left(P, P_{(-i)}\right)$ considerando uma amostra de tamanho $Q$ da distribuição a posteriori de $p(\gamma \mid \mathcal{D})$ é dada por

$$
K\left(\widehat{P, P_{(-i)}}\right)=-\log \left(\widehat{C P O_{i}}\right)+\frac{1}{Q} \sum_{q=1}^{Q} \log \left[g\left(t_{i} \mid \boldsymbol{\gamma}_{q}\right)\right]
$$

sendo $\left(\widehat{C P O_{i}}\right)$ como descrito na Equação (1.2).

Segundo McCulloch (1989) e Cho et al. (2009), a calibração de $K\left(P, P_{(-i)}\right)$ pode ser efetuada resolvendo em $p_{i}$ a equação

$$
K\left(P, P_{(-i)}\right)=K\left(B(0,5), B\left(p_{i}\right)\right)=-\frac{\log \left\{4 p_{i}\left(1-p_{i}\right)\right\}}{2}
$$

em que $B(p)$ denota uma distribuição de Bernoulli com probabilidade de sucesso $p$. Isto implica que descrever resultados usando $\pi\left(\gamma \mid \mathcal{D}_{(-i)}\right)$ ao invés de $p(\gamma \mid \mathcal{D})$ é equivalente com a descrição de um evento não observado com probabilidade $p_{i}$ quando a probabilidade correta é 0,5 . Após o cálculo de $K\left(P, P_{(-i)}\right)$ em (1.5) a solução em $p_{i}$ da Equação (1.7) é calculada por $p_{i}=0,5\left[1+\sqrt{1-\exp \left\{-2 K\left(P, P_{(-i)}\right)\right\}}\right]$. Isto implica que $0,5 \leq p_{i} \leq 1$. Além disso, se $p_{i} \gg 0,5$ implica que o $i$-ésimo caso é influente.

Neste trabalho analisamos modelos de sobrevivência sem e com fração de cura e ainda modelos destrutivos com fração de cura com o intuito de analisar qual proporciona um melhor ajuste. São propostas análise de influência caso a caso para investigar possíveis problemas com o modelo ajustado. O estudo é feito com enfoque bayesiano. São apresentados estudos de simulação e estudos com dados reais. A proposta deste trabalho é interessante uma vez que não encontramos na literatura análises de diagnósticos para os 
modelos aqui discutidos.

\subsection{Organização dos capítulos}

No Capítulo 2 desenvolvemos métodos bayesianos via Monte Carlo em cadeias de Markov (MCMC) para um modelo de regressão exponencial por partes e realizamos um estudo de diagnóstico. No Capítulo 3 abordamos modelos de sobrevivência com fração de cura, fazemos uma análise de sensibilidade na estimativa dos parâmetros a posteriori para um conjunto de dados reais propiciando a escolha de um modelo final com melhor ajuste aos dados. No Capítulo 4 tratamos de um modelo destrutivo com fração de cura baseado na distribuição binomial negativa, neste modelo temos a inserção de um parâmetro $(p)$ que permite estimar a probabilidade do número de causas não destruídas, também realizamos um estudo de diagnóstico considerando um conjunto de dados de melanoma. Finalmente, no Capítulo 5 apresentamos as conclusões com base nos resultados obtidos e as propostas futuras. 


\section{Capítulo 2}

\section{Modelo Exponencial por Partes}

Segundo Ibrahim et al. (2001), o MEP é um dos modelos mais populares utilizados em análise de sobrevivência, a popularidade e importância se deve ao fato deste modelo ser capaz de acomodar funções de taxa de falha com diversas formas, não havendo a necessidade de impormos restrições quanto a forma da função de risco para obtermos um ajuste apropriado do modelo aos dados, como acontecem com alguns modelos como por exemplo, com o modelo exponencial e Weibull. Esta característica torna o modelo bastante flexível. Uma das dificuldades em se trabalhar com o modelo exponencial por partes está em definir a partição do eixo dos tempos a ser utilizada. Em geral, tal partição é escolhida arbitrariamente como discutido por Gamerman (1994), Barbosa et al. (1996), (Ibrahim et al., 2001), através de algum critério de seleção de modelo (Yin, 2005) ou aleatoriamente (Demarqui et al., 2008).

\subsection{Formulação do modelo}

Seja $T$ uma variável aleatória não-negativa representando o tempo até a ocorrência de um evento de interesse, denominado tempo de falha. Considere uma partição finita e arbitrária do eixo dos tempos, tal que, $s_{0}<s_{1}<\ldots<s_{J}<\infty$, com $s_{0}=0$ e $s_{J}>t$, para algum $t$ observado, com $t>0$, admita que tal partição divida o eixo do tempo em $J$ 
intervalos disjuntos, denotados por $I_{1}=\left(s_{0}, s_{1}\right], I_{2}=\left(s_{1}, s_{2}\right], \ldots, I_{J}=\left(s_{J-1}, s_{J}\right]$ (Ibrahim et al., 2001).

O MEP, algumas vezes chamado modelo semiparamétrico é caracterizado pela aproximação da função taxa de falha, $h(t)$ por segmentos de retas definidos pelos intervalos determinados pela partição $\left\{s_{0}, \ldots, s_{J}\right\}$, isto é, assume-se que em cada intervalo $I_{j}=$ $\left(s_{j-1}, s_{j}\right], j=1, \ldots, J$ a função taxa de falha é constante e denotada por $h(t)=\lambda_{j}, \lambda_{j}>0$, $\forall t \in I_{j}$. Consequentemente, a função taxa acumulada, $H(t)$, associada ao $j$-ésimo intervalo, $I_{j}=\left(s_{j-1}, s_{j}\right]$, é dada pela soma das áreas dos retângulos cujas bases são determinadas pelos intervalos definidos pela partição $\left\{s_{0}, \ldots, s_{J}\right\}$, e com alturas dada pela função taxa de falha, $h(t)$, ou seja, $H(t)=\sum_{k=1}^{j-1} \lambda_{k}\left(s_{k}-s_{k-1}\right)+\lambda_{j}\left(t-s_{j-1}\right)$, para $t \in I_{j}, j=1, \ldots, J$.

Temos que a função densidade de probabilidade e a função de sobrevivência do MEP são expressas respectivamentes por

$$
f(t \mid \boldsymbol{\lambda})=\left\{\begin{array}{l}
\lambda_{1} \exp \left\{-\lambda_{1} t\right\}, \text { se } t \in I_{1} \\
\lambda_{j} \exp \left\{-\left[\sum_{k=1}^{j-1} \lambda_{k}\left(s_{k}-s_{k-1}\right)+\lambda_{j}\left(t-s_{j-1}\right)\right]\right\}, \text { se } t \in I_{j}, j>1
\end{array}\right.
$$

e

$$
S(t \mid \boldsymbol{\lambda})=\left\{\begin{array}{l}
\exp \left\{-\lambda_{1} t\right\}, \text { se } t \in I_{1} \\
\exp \left\{-\left[\sum_{k=1}^{j-1} \lambda_{k}\left(s_{k}-s_{k-1}\right)+\lambda_{j}\left(t-s_{j-1}\right)\right]\right\}, \text { se } t \in I_{j}, j>1
\end{array}\right.
$$

$\operatorname{com} \boldsymbol{\lambda}=\left(\lambda_{1}, \lambda_{2}, \ldots, \lambda_{J}\right), \lambda_{j}>0, \forall j=1, \ldots, J$

Apresentamos na Figura 2.1 os gráficos da função de sobrevivência e da função taxa de falha para o MEP. Os mesmos foram gerados considerando quatro partições no eixo dos tempos $(J=4)$ e com função de taxa de falha assumindo os seguintes valores $\lambda_{1}=0,017 ; \lambda_{2}=0,043 ; \lambda_{3}=0,120$ e $\lambda_{4}=0,760$. 
(a)

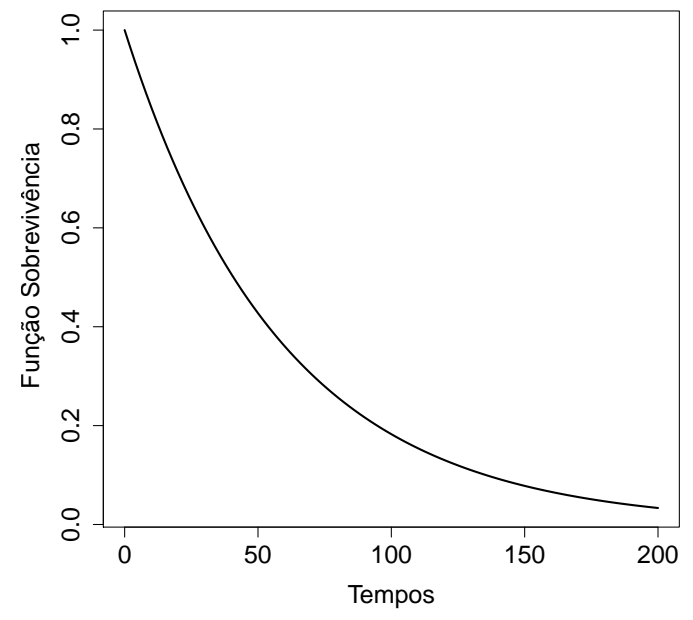

(b)

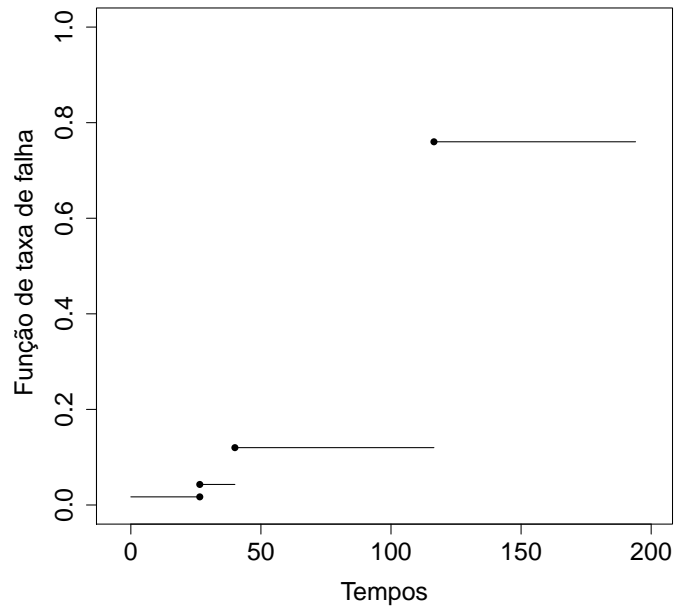

FIGURA 2.1: (a) Função de sobrevivência e (b) função de taxa de falha para o MEP.

\subsection{Modelo de regressão semiparamétrico (MRSP)}

Em muitas situações práticas temos que o tempo de vida $(T)$ é influenciado por uma ou mais covariáveis. Por exemplo, o tempo de sobrevivência de um paciente pode depender de sua idade, da quantidade de cigarros que fuma por dia e de uma série de outros fatores. Uma maneira de determinar o relacionamento entre o tempo de vida e o conjunto de covariáveis é por meio de um modelo de regressão. Podemos citar duas classes importantes de modelos de regressão: os modelos de riscos proporcionais para $T$ e modelos de locação-escala ou modelos de testes acelerados para $\log (T)$. Abordaremos neste trabalho apenas a primeira classe. Uma descrição detalhada sobre modelos de testes acelerados pode ser obtida em Lawless \& Lawless (1982) e Nelson (1990). A família de modelos de riscos proporcionais é largamente utilizada na maioria das vezes que tratamos de dados sobrevivência e pode ser definida pela função de risco como

$$
h(t ; \boldsymbol{x})=h_{0}(t) \exp \left\{\boldsymbol{x}^{\top} \boldsymbol{\beta}\right\}
$$

em que, $\boldsymbol{\beta}$ é um vetor $p \times 1$ de coeficientes de regressão associados ao vetor de covariáveis $\boldsymbol{x}^{\top}=\left(x_{1}, x_{2}, \ldots, x_{p}\right)$ e $h_{0}$ é a função de risco para um indivíduo, também chamada de 
função basal.

A principal suposição considerada para este modelo é a proporcionalidade entre os riscos. Se não assumirmos uma particular forma para $h_{0}(t)$, obtemos o modelo de riscos proporcionais de Cox (Cox, 1972), que tem ampla literatura de aplicações e extensões (Lawless \& Lawless, 1982). Uma outra abordagem é obtida se considerarmos uma representação paramétrica para $h_{0}(t)$. Desta forma obtemos uma família paramétrica de riscos proporcionais. Considerando a formulação do modelo como apresentada na Seção (2.1) e assumindo a função de risco $h_{j}(t)=\lambda_{j} \exp \left(\boldsymbol{x}^{\top} \boldsymbol{\beta}\right)$, podemos expressar a função densidade de probabilidade para o MRSP por

$$
f(t ; \boldsymbol{\gamma})=\left\{\begin{array}{l}
\lambda_{1} \exp \left(\boldsymbol{x}^{\top} \boldsymbol{\beta}\right) \exp \left\{-\lambda_{1} t \exp \left(\boldsymbol{x}^{\top} \boldsymbol{\beta}\right)\right\}, \text { se } t \in I_{1} \\
\lambda_{j} \exp \left(\boldsymbol{x}^{\top} \boldsymbol{\beta}\right) \exp \left\{-\left[\sum_{k=1}^{j-1} \lambda_{k}\left(s_{k}-s_{k-1}\right)+\lambda_{j}\left(t-s_{j-1}\right)\right] \exp \left\{\boldsymbol{x}^{\top} \boldsymbol{\beta}\right\}\right\} \\
\text { se } t \in I_{j}, j>1
\end{array}\right.
$$

A partir da equação (2.4) podemos mostrar que a função de sobrevivência do modelo é dada por

$$
S(t ; \boldsymbol{\gamma})=\left\{\begin{array}{l}
\exp \left\{-\lambda_{1} t \exp \left(\boldsymbol{x}^{\top} \boldsymbol{\beta}\right)\right\}, \text { se } t \in I_{1} \\
\exp \left\{-\left[\sum_{k=1}^{j-1} \lambda_{k}\left(s_{k}-s_{k-1}\right)+\lambda_{j}\left(t-s_{j-1}\right)\right] \exp \left\{\boldsymbol{x}^{\top} \boldsymbol{\beta}\right\}\right\}, \text { se } t \in I_{j}, j>1
\end{array}\right.
$$

$\operatorname{com} \boldsymbol{\gamma}=(\boldsymbol{\lambda}, \boldsymbol{\beta}), \lambda_{j}>0, \forall j=1, \ldots, J$

\subsection{Inferência bayesiana}

Denotamos por $\boldsymbol{D}=(n, \mathbf{t}, \boldsymbol{X}, \delta)$ os dados observados, com $\boldsymbol{t}=\left(t_{1}, t_{2}, \ldots, t_{n}\right)^{\top}$, $\boldsymbol{\delta}=\left(\delta_{1}, \delta_{2}, \ldots, \delta_{n}\right)^{\top}, \operatorname{com} \delta_{i}=1$ se o $i$-ésimo indivíduo for falha e 0 caso contrário. $\boldsymbol{X}$ é uma matriz $n \times p$ de covariáveis. Assumindo $\boldsymbol{\lambda}=\left(\lambda_{1}, \lambda_{2}, \ldots, \lambda_{j}\right)^{\top}$, podemos escrever a 
função de verossimilhança de $\boldsymbol{\gamma}=(\boldsymbol{\beta}, \boldsymbol{\lambda})$ para $n$ indivíduos por

$$
\begin{aligned}
L(\boldsymbol{\gamma} ; \boldsymbol{D})= & \prod_{i=1}^{n} \prod_{j=1}^{J}\left(\lambda_{j} \exp \left(\boldsymbol{x}_{\boldsymbol{i}}^{\top} \boldsymbol{\beta}\right)\right)^{\nu_{i j} \delta_{i}} \exp \left\{-\nu_{i j}\left[\lambda_{j}\left(t_{i}-s_{j-1}\right)\right.\right. \\
& \left.\left.+\sum_{k=1}^{j-1} \lambda_{k}\left(s_{k}-s_{k-1}\right)\right] \exp \left(\boldsymbol{x}_{\boldsymbol{i}}^{\top} \boldsymbol{\beta}\right)\right\} .
\end{aligned}
$$

sendo que $\nu_{i j}=1$ indica se o $i$-ésimo indivíduo é falha ou censura no $j$-ésimo intervalo e 0 caso contrário, $\boldsymbol{x}_{i}^{\top}$ denota o vetor $p \times 1$ de covariáveis para o $i$-ésimo indivíduo e $\boldsymbol{\beta}=\left(\beta_{1}, \beta_{2}, \ldots, \beta_{p}\right)^{\top}$ é o vetor dos coeficientes de regressão correspondentes. O indicador $\nu_{i j}$ é necessário para definir a função verossimilhança sobre cada um dos $J$ intervalos. Realizamos a partição do eixos dos tempos como sugerido por Yin (2005) que afirma que uma forma razoável de partição seria equilibrar o número de falhas nos intervalos, além de garantir que pelo menos uma falha aconteça em cada intervalo de tempo.

Assumindo independência entres os parâmetros do MRSP a densidade a priori conjunta é expressa por $\pi(\boldsymbol{\beta}, \boldsymbol{\lambda})=\pi(\boldsymbol{\beta}) \pi(\boldsymbol{\lambda})$ em que os parâmetros $\boldsymbol{\beta}_{\boldsymbol{k}}$ seguem uma distribuição Normal, com média $\mu_{\beta_{k}}$ e variância $\sigma_{\beta_{k}}^{2}$, em notação $\boldsymbol{\beta}_{\boldsymbol{k}} \sim N\left(\mu_{\beta_{k}}, \sigma_{\beta_{k}}^{2}\right), k=1, \ldots, p$ e $\lambda_{j} \sim \pi\left(\lambda_{j}\right), j=1, \ldots, J$. Combinando essa densidade a priori com a verossimilhança (2.6), a densidade a posteriori conjunta é dada por

$$
\begin{aligned}
\pi(\boldsymbol{\beta}, \boldsymbol{\lambda} \mid \boldsymbol{D}) & =L(\boldsymbol{\beta}, \boldsymbol{\lambda} ; \boldsymbol{D}) \pi(\boldsymbol{\beta}) \pi(\boldsymbol{\lambda}) \\
& \propto \prod_{i=1}^{n} \prod_{j=1}^{J}\left(\lambda_{j} \exp \left(\boldsymbol{x}_{\boldsymbol{i}}^{\top} \boldsymbol{\beta}\right)\right)^{\nu_{i j} \delta_{i}} \exp \left\{-\nu_{i j}\left[\lambda_{j}\left(t_{i}-s_{j-1}\right)\right.\right. \\
& \left.\left.+\sum_{k=1}^{j-1} \lambda_{k}\left(s_{k}-s_{k-1}\right)\right] \exp \left(\boldsymbol{x}_{\boldsymbol{i}}^{\top} \boldsymbol{\beta}\right)\right\} \exp \left\{\frac{\boldsymbol{\beta}(2 \mu-\boldsymbol{\beta})}{2 \sigma^{2}}\right\} \pi(\boldsymbol{\lambda}),
\end{aligned}
$$

em que $\pi(\boldsymbol{\lambda})$ é uma distribuição a priori de $\lambda$.

Como a densidade a posteriori conjunta não é uma densidade padrão usamos métodos de Monte Carlo via cadeias de Markov (MCMC), tais como o amostrador de Gibbs e Metropolis-Hasting. Podemos mostrar que as densidades a posteriori condicionais para o amostrador de Gibbs são expressas por $\pi(\boldsymbol{\beta} \mid \boldsymbol{\lambda}, \boldsymbol{D}) \propto L(\boldsymbol{\beta}, \boldsymbol{\lambda} ; \boldsymbol{D}) \pi(\boldsymbol{\beta})$ e $\pi(\boldsymbol{\lambda} \mid \boldsymbol{\beta}, \boldsymbol{D}) \propto$ $L(\boldsymbol{\beta}, \boldsymbol{\lambda} ; \boldsymbol{D}) \pi(\boldsymbol{\lambda})$. 
Como as densidades a posteriori condicionais não possuem forma fechada usaremos o algoritmo de Metropolis-Hastings dentro do ciclo do algoritmo de Gibbs (Gilks et al., 1996) para gerar amostras de $\boldsymbol{\beta}$ e $\boldsymbol{\lambda}$.

\subsection{Aplicação}

Nesta Seção ilustraremos a metodologia descrita anteriormente com um conjunto de dados simulados e um conjunto de dados reais.

\subsubsection{Dados simulados}

Para examinar o desempenho das medidas de diagnósticos propostas neste trabalho, nós consideramos um conjunto de dados simulados. Consideramos as covariáveis $x_{i 1}$ e $x_{i 2}, i=1, \ldots n$, sendo $x_{i 1}$ gerado de uma distribuição Bernoulli com parâmetro $p=0,5$ e $x_{i 2}$ de uma distribuição normal $(0,2)$. Os tempos de falha $T_{i}$ foram gerados de uma distribuição exponencial $\left(\lambda_{i}\right)$ com função de $\operatorname{risco} h\left(t_{i}\right)=\lambda_{1} \exp \left(\beta_{1} x_{i 1}+\beta_{2} x_{i 2}\right)$ em que, $\beta_{1}=0,8, \beta_{2}=1,2$ e $\lambda_{1}=0,6$; os tempos de censura $C_{i}$ também foram gerados de uma distribuição exponencial com parâmetro $\lambda_{c}$, em que o mesmo é escolhido de forma a monitorar a quantidade de dados censurados, neste caso temos $16 \%$ de dados censurados. Assumimos ainda, que $T_{i}$ e $C_{i}$ são independentes. Os tempos de sobrevivência $\mathrm{t}_{i}, i=1, \ldots 50$, foram tomados como $t_{i}=\min \left(T_{i}, C_{i}\right), \delta_{i}$ é o indicador de censuras com $\delta_{i}=1$, se $T_{i} \leq C_{i}$, e 0 , se $T_{i}>C_{i}$. Selecionamos os casos 13, 28 e 39 para perturbar afim de criarmos observações influentes no conjunto de dados. Os casos perturbados 13 e 39 representam tempos de falha e o caso 28 tempo de censura. Nós escolhemos um, dois ou três casos e perturbamos a variável resposta da seguinte maneira $\tilde{t}_{i}=t_{i}+5 \tau_{t}$, em que $\tau_{t}$ são os desvios padrão dos tempos $t_{i}$. Na Tabela 2.1 o conjunto de dados $(a)$ denota os dados originais simulados sem nenhuma perturbação e os conjuntos de dados $(b)-(f)$ denota os dados com casos perturbados.

Consideramos uma análise bayesiana com densidades a priori independentes para 
os parâmetros do modelo, com $\beta_{k} \sim N(0,100), k=1,2$, e $\lambda_{j} \sim G(1 ; 0,0001)$, com $j=1$ em que, $N(0,100)$ denota uma distribuição normal com média 0 e variância 100 e $G(1 ; 0,0001)$ uma distribuição gama com média 1/0,0001 e variância $1 /(0,0001)^{2}$. A distribuição a posteriori para os parâmetros obtida não é tratável analiticamente, portanto métodos MCMC podem ser considerados. Como as condicionais não tem forma fechada geramos amostras das distribuição a posteriori dos parâmetros usando o algoritmos de Gibbs com Metropolis-Hasting da seguinte forma: geramos duas cadeias paralelas cada uma com 35.000 iterações para cada parâmetro, descartamos as primeiras 5.000 iterações afim de eliminar os efeitos dos valores iniciais e evitar problemas de correlação, consideramos espaçamento de tamanho 10, resultando uma amostra de Gibbs de tamanho 6.000. Para monitorar a convergência do amostrador de Gibbs utilizamos a aproximação desenvolvida por Gelman e Rubin(1992). Afim de avaliar a robustez do modelo relacionado às escolhas dos hiperparâmetros das priori, um estudo de sensibilidade foi realizado, no qual constatamos que as estimativas dos parâmetros a posteriori não apresentaram diferenças significativas. Na Tabela 2.1 apresentamos as estimativas a posteriori para os dados simulados com e sem perturbações em alguns casos e os valores para os critérios de seleção $B$ e $D I C$ (discutidos na Seção 1.1). Para o cálculo da CPO (Equação 1.1), temos que, se $\delta_{i}=1$ então $g\left(t_{i} \mid \gamma\right)=f(t ; \gamma)($ Equação 2.4$)$ e se $\delta_{i}=0$ temos $g\left(t_{i} \mid \gamma\right)=S(t ; \gamma)$ (Equação 2.5). Os critérios $B$ e $D I C$ demonstram que o modelo sem casos perturbados (a) apresenta o melhor ajuste para os dados.

TABELA 2.1: Médias e desvios padrão a posteriori para os parâmetros do modelo dos dados simulados.

\begin{tabular}{cccccccccc}
\hline Dados & Casos & \multicolumn{2}{c}{$\beta_{1}$} & \multicolumn{2}{c}{$\beta_{2}$} & \multicolumn{2}{c}{$\lambda_{1}$} & B & DIC \\
& perturbados & Média & DP. & Média & DP. & Média & DP. & & \\
\hline a & Nenhum & 0,747 & 0,327 & 1,002 & 0,079 & 0,516 & 0,136 & $-66,410$ & 132,463 \\
b & 13 & $-0,009$ & 0,468 & 0,360 & 0,109 & 0,179 & 0,066 & $-135,895$ & 248,703 \\
c & 28 & $-0,487$ & 0,453 & 0,616 & 0,126 & 0,308 & 0,110 & $-130,559$ & 236,797 \\
d & 39 & 2,384 & 0,336 & 0,810 & 0,105 & 0,091 & 0,023 & $-98,637$ & 180,818 \\
e & $\{13,28\}$ & $-0,353$ & 0,513 & 0,305 & 0,129 & 0,160 & 0,065 & $-146,771$ & 276,849 \\
f & $\{13,28,39\}$ & 0,792 & 0,351 & 0,062 & 0,089 & 0,055 & 0,015 & $-152,808$ & 291,991 \\
\hline
\end{tabular}

Considerando as amostras de Gibbs, estimamos as medidas de divergência de $\mathrm{K}-\mathrm{L}$ (apresentado na Seção 1.2.1) para cada um dos casos (a,b,c,d,e e f), esses resultados são 
graficados na Figura 2.2. Ainda estimamos as medidas de calibração (discutidos na Seção 1.2.1) referentes a cada caso. Os resultados apresentados na Tabela 2.2 indicam que sem perturbação nos dados $(a)$, os casos selecionados não são influentes, pois obtivemos valores pequenos para $K\left(P, P_{(-i)}\right)$ em cada caso. Entretanto, após perturbação $(b-f)$ obtivemos medidas $K\left(P, P_{(-i)}\right)$ grandes e calibração próximas ou iguais ao valor 1 indicando que estes casos são influentes.

TABELA 2.2: Medidas de diagnósticos para os dados simulados.

\begin{tabular}{cccc}
\hline Nomes dos Dados & Identificação dos casos & $K\left(P, P_{(-i)}\right)$ & Calibração \\
\hline a & 13 & 0,011 & 0,574 \\
& 28 & 0,262 & 0,819 \\
& 39 & 0,001 & 0,530 \\
b & 13 & 10,496 & 1,000 \\
c & 28 & 11,267 & 1,000 \\
d & 39 & 8,174 & 1,000 \\
e & 13 & 3,813 & 0,999 \\
& 28 & 2,932 & 0,999 \\
f & 13 & 2,129 & 0,996 \\
& 28 & 2,563 & 0,998 \\
& 39 & 1,975 & 0,995 \\
\hline
\end{tabular}

Na Figura 2.2 plotamos $K\left(P, P_{(-i)}\right)$ para o modelo proposto, podemos notar que K-L identifica os casos influentes apresentando valores maiores de $K\left(P, P_{(-i)}\right)$ para os casos perturbados se comparados aos outros casos.

(a)

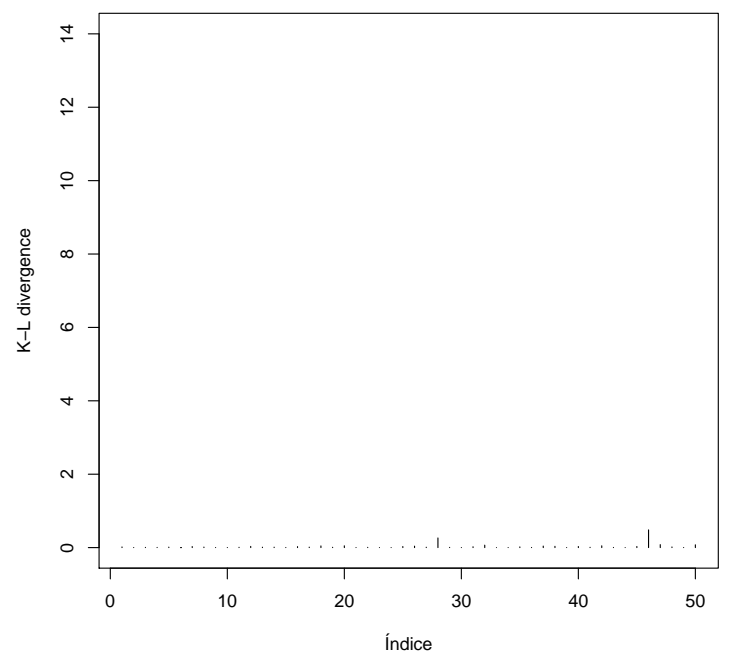

(b)

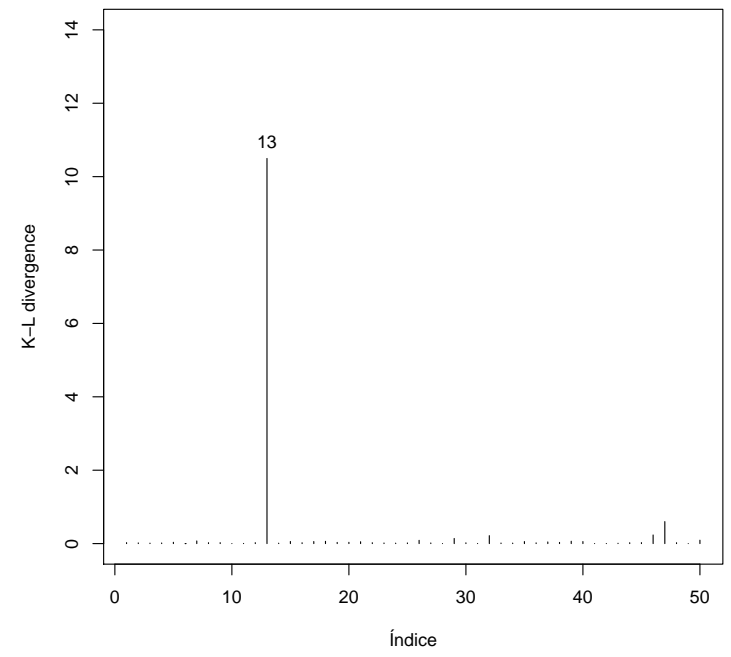


(c)

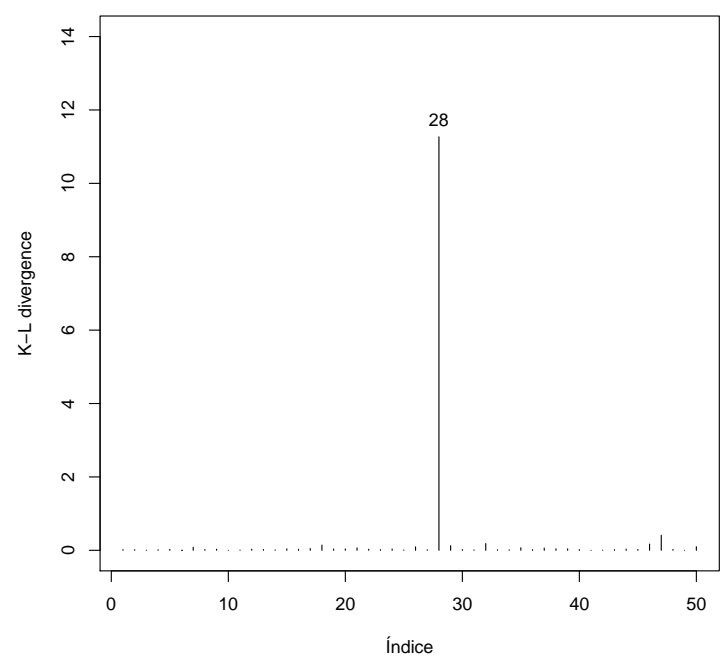

(e)

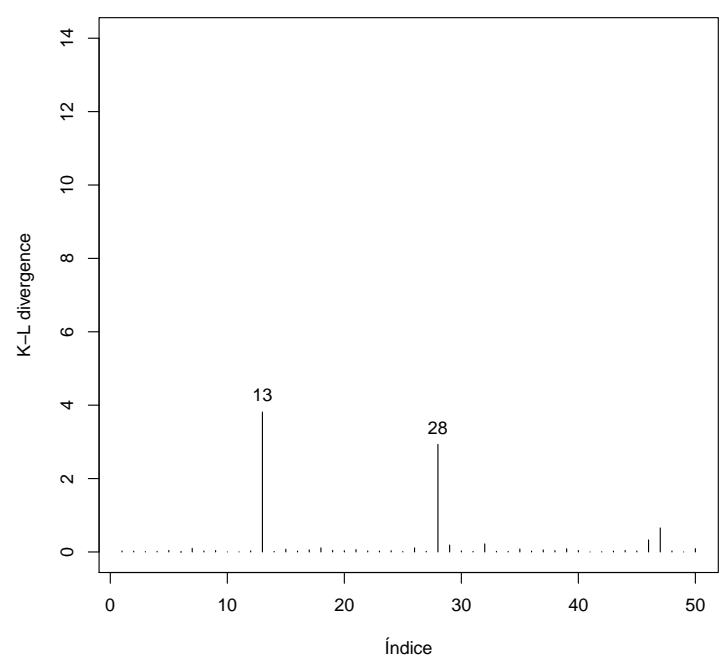

(d)

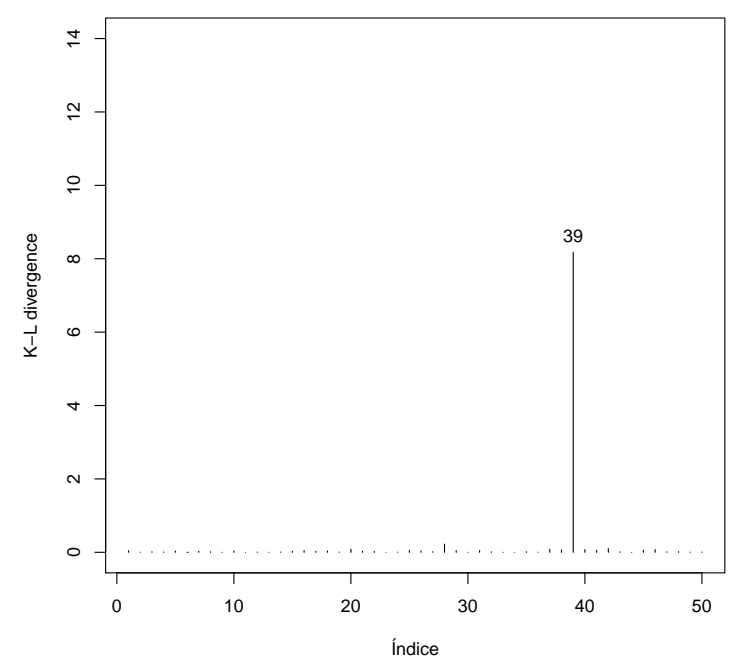

(f)

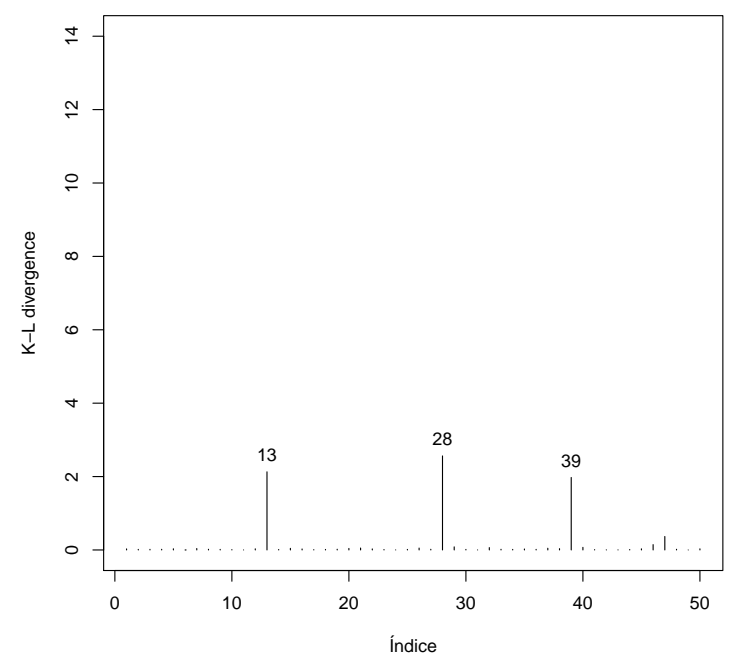

FIGURA 2.2: Gráfico de índices de $K\left(P, P_{(-i)}\right)$ para os dados simulados.

\subsubsection{Dados de infecção renal}

Os dados considerados nesta seção referem-se ao estudo descrito em McGilchrist \& Aisbett (1991), os quais relacionam os tempos de reincidência de infecção renal em 38 pacientes. Consideramos a presença de cinco covariáveis: $x_{1}$, idade do paciente (com média de 43,7 anos e desvio padrão de 14,8 anos); $x_{2}$, gênero do paciente (0-masculino, 1-feminino); $x_{3}, x_{4}$ e $x_{5}$ indicam o tipo da doença apresentada pelo paciente, sendo GN (glomerulo nefrite), AN (nefrite aguda) e PKD (rim policístico) respectivamente. Em nosso estudo consideramos somente o primeiro tempo (em dias) observado. Há no conjunto de 
dados $28,9 \%$ de censuras.

Pelo método gráfico baseado no tempo total em teste (TTT) transformado, descrito por Barlow \& Campo (1975) identificamos a forma da função de risco com o objetivo de avaliar se o modelo é adequado aos tempos de vida. Em nosso caso, temos indício de que os dados apresentam a função de risco não monótona, como observado na Figura 2.3, assim ajustamos MRSP (descrito na Seção 2.2), considerando $J=1,2,3,4$ e 5 com as cinco covariáveis descritas anteriormente.

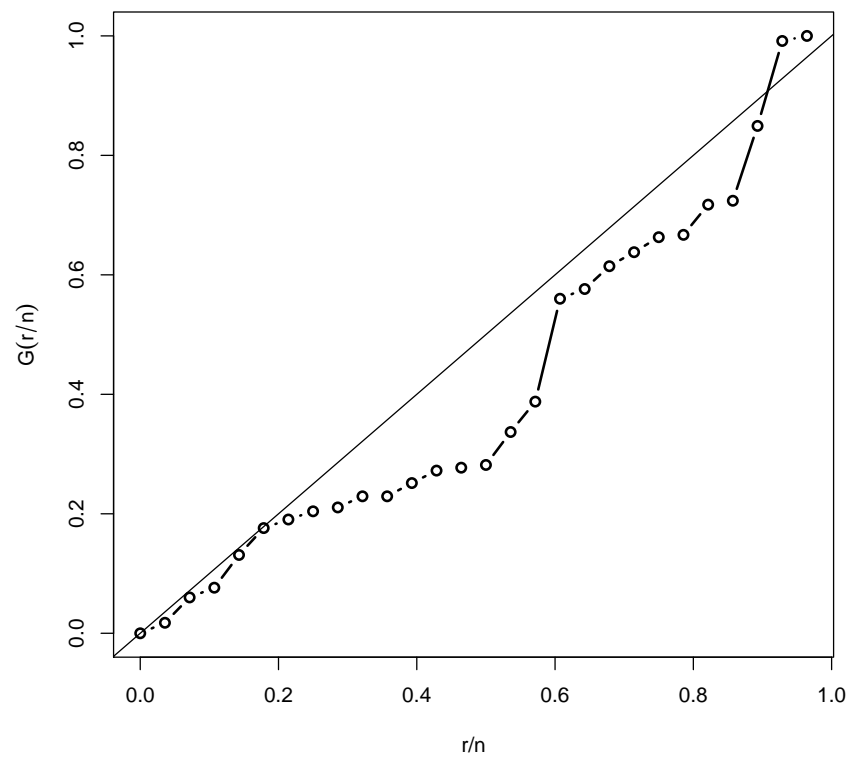

FIGURA 2.3: Gráfico TTT plot para dados de infecção renal.

Para nossa análise bayesiana consideramos densidades a priori independentes para os parâmetros $\beta_{k}$, ou seja, $\beta_{k} \sim N(0 ; 100), k=1, \ldots 5$ e escolhemos para $\lambda$ densidades a priori dependentes, por existir uma relação de dependência entre os parâmetros em cada partição, com a seguinte distribuição, $\phi_{1} \sim G(0,1 ; 0,01), \xi \sim G(0,1 ; 0,01)$ e $\phi_{j} \sim N\left(\phi_{j-1} ; \xi\right)$, $j=2, \ldots$ J. Como os parâmetros $\lambda_{j}$ são não negativos, adotamos a seguinte parametrização, $\lambda_{j}=\log \left(\phi_{j}\right)$, com $j=1, \ldots$ J. Considerando essas densidades a priori geramos amostras de Gibbs através do algoritmo de Gibbs com Metropolis-Hasting da seguinte forma: geramos duas cadeias paralelas cada uma com 35.000 iterações com um burn in de 5.000 e saltos de tamanho 10, resultando uma amostra de Gibbs de tamanho 6.000. Para monitorar a 
convergência do amostrador de Gibbs utilizamos a aproximação desenvolvida por Gelman e Rubin(1992). Na Tabela 2.3 apresentamos os valores para os critérios de seleção $B$ e $D I C$ para os modelos ajustados. Ambos critérios indicam um MRSP com $J=4$ intervalos disjuntos na partição do eixo dos tempos. Para avaliar a robustez do modelo relacionado às escolhas dos hiperparâmetros das priori, um estudo de sensibilidade foi realizado, sendo que as estimativas dos parâmetros a posteriori não apresentaram diferenças significativas e não alteraram os resultados da Tabela 2.3 e Tabela 2.4 .

TABELA 2.3: Critérios de seleção de modelos considerando os dados de infecção renal.

\begin{tabular}{ccc}
\hline Modelo & \multicolumn{2}{c}{ Critério } \\
$\mathrm{J}$ & $B$ & $D I C$ \\
\hline 1 & $-160,677$ & 318,500 \\
2 & $-163,058$ & 319,500 \\
3 & $-162,515$ & 320,500 \\
4 & $-160,619$ & 317,000 \\
5 & $-164,677$ & 323,200 \\
\hline
\end{tabular}

Na Tabela 2.4 apresentamos os resumos da distribuição a posteriori do MRSP para o melhor modelo. Para todos os parâmetros observamos valores de $\hat{R}$ próximos de 1, indicando que as iterações foram suficientes para se chegar a convergência. A "highest posterior density" (HPD)(Chen \& Shao, 1999) (maiores detalhes consulte Bernardo \& Smith, 2000) com 95\% de credibilidade indica que somente as covariáveis sexo e PKD são significativas.

TABELA 2.4: Resumos a posteriori para os tempos.

\begin{tabular}{ccccc}
\hline Parâmetro & Média & Desvio Padrão & $H P D(95 \%)$ & $\hat{R}$ \\
\hline$\beta_{1 \text { (idade) }}$ & $-0,001$ & 0,016 & $(-0,034 ; 0,028)$ & 1,001 \\
$\beta_{2 \text { (sexo) }}$ & $-1,175$ & 0,484 & $(-2,100 ;-0,195)$ & 1,003 \\
$\beta_{3(\mathrm{GN})}$ & 0,251 & 0,596 & $(-0,944 ; 1,391)$ & 1,001 \\
$\beta_{4(\mathrm{AN})}$ & 0,782 & 0,628 & $(-0,373 ; 2,077)$ & 1,001 \\
$\beta_{5(\mathrm{PKD})}$ & $-2,028$ & 1,016 & $(-4,135 ;-0,169)$ & 1,006 \\
$\lambda_{1}$ & 0,020 & 0,017 & $(0,001 ; 0,053)$ & 1,001 \\
$\lambda_{2}$ & 0,051 & 0,044 & $(0,003 ; 0,135)$ & 1,002 \\
$\lambda_{3}$ & 0,014 & 0,015 & $(0,000 ; 0,041)$ & 1,001 \\
$\lambda_{4}$ & 0,030 & 0,027 & $(0,001 ; 0,076)$ & 1,002 \\
\hline
\end{tabular}

Com as amostras de Gibbs estimamos as medidas de divergência de K-L para cada uma das observações, esses resultados são graficados na Figura 2.4, em que as observações 10, 15 e 21 apresentam maiores valores quando comparados com as demais observações. 
Para verificarmos se essas observações são influentes estimamos a calibração da medida de divergência K-L, essas estimativas são apresentados na Tabela 2.5 conjuntamente com as respectivas estimativas da divergência $\mathrm{K}-\mathrm{L}$, indicando que as três observações são influentes.

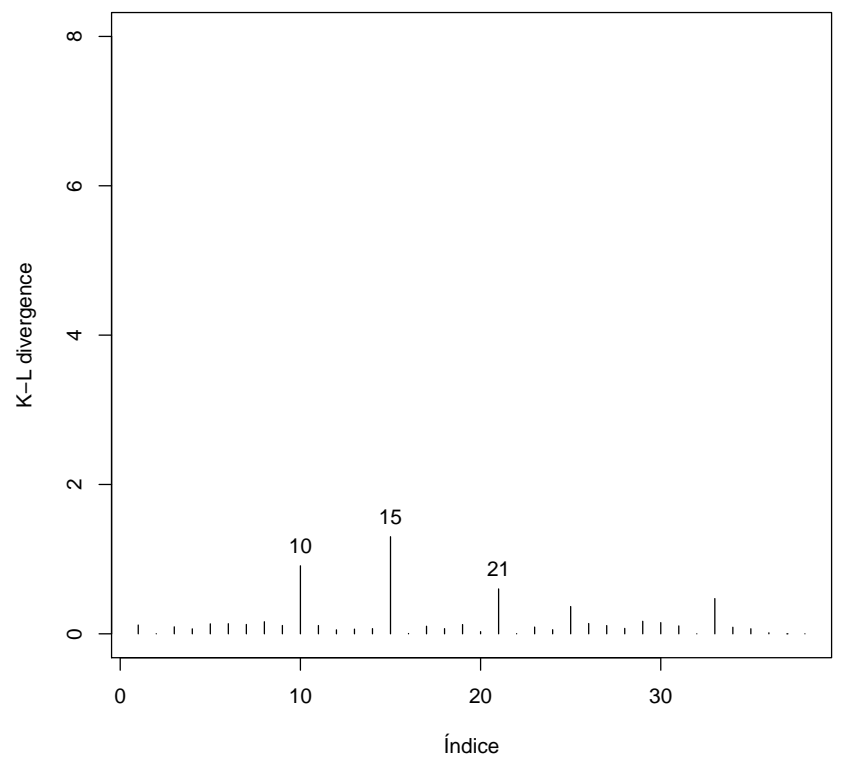

FIGURA 2.4: Gráfico de índices de $K\left(P, P_{-i}\right)$ para os dados de infecção renal.

TABELA 2.5: Identificação dos casos influentes para dados de infecção renal.

\begin{tabular}{rcccc}
\hline \multicolumn{3}{c}{ Identificação do caso } & \multicolumn{2}{c}{ Influência caso a caso } \\
\hline Paciente & Tempo & Idade & $K\left(P, P_{(-i)}\right)$ & Calibração \\
\hline 10 & 154 & 51,500 & 0,910 & 0,957 \\
15 & 536 & 17,000 & 1,300 & 0,981 \\
21 & 562 & 46,500 & 0,601 & 0,918 \\
\hline
\end{tabular}

Na Tabela 2.6 apresentamos as novas estimativas dos parâmetros após a exclusão de cada caso influente e também as variações relativas $(V R)$ nas estimativas após termos excluídos um dos três pontos influentes ou descartados todos de uma só vez (representados pelo conjunto $I=\{10,15,21\}$ ). As $V R$ (em porcentagem) para cada parâmetro estimado são obtidas por $V R_{\gamma_{d}}=\left|\left(\hat{\gamma}_{d}-\hat{\gamma}_{d(I)}\right) / \hat{\gamma}_{d}\right| \times 100 \%$, em que $\hat{\gamma}_{d(I)}$ denota a média a posteriori de $\gamma_{d}$, com $d=1, \ldots, 9$ após o conjunto $I$ de observações ter sido removido. A densidade a posteriori máxima (HPD) com $95 \%$ de credibilidade para cada nova estimativa é 
apresentada entre parênteses na Tabela 2.6.

Notamos valores altos de $V R$ para o parâmetro $\widehat{\beta}_{1}$ quando há exclusão de qualquer um dos pontos influentes e também quando excluímos o conjunto $I$, observamos que a estimação dos parâmetros do modelo apresenta sensibilidade em relação aos pontos influentes, observando-se a ocorrência de mudança de sinal no valor do parâmetro como ocorre em $\widehat{\beta}_{1}$ quando excluímos o caso 10. Em adição nós notamos que ocorreram algumas mudanças nas estimativas de alguns coeficientes, particularmente $\widehat{\beta}_{5}$ deixa de ser significativo após retirada do caso 21 e do conjunto $I$ de observações influentes.

TABELA 2.6: Estimativas, $V R$ (em \%) e a correspondente HPD (95\%) ajustados para o conjunto de dados de infecção renal.

\begin{tabular}{ccccc}
\hline Parâmetros & \multicolumn{4}{c}{ Observações descartadas } \\
& 10 & 15 & 21 & $I$ \\
\hline \multirow{3}{*}{$\beta_{1 \text { (idade) }}$} & 0,006 & $-0,018$ & $-0,004$ & $-0,008$ \\
& 464 & 970 & 135 & 370 \\
& $(-0,023 ; 0,039)$ & $(-0,048 ; 0,015)$ & $(-0,037 ; 0,029)$ & $(-0,037 ; 0,025)$ \\
\hline \multirow{3}{*}{$\beta_{2 \text { (sexo) }}$} & $-1,938$ & $-1,137$ & $-1,400$ & $-2,188$ \\
& 64 & 03 & 19 & 86 \\
& $(-3,114 ;-0,815)$ & $(-2,007 ;-0,178)$ & $(-2,318 ;-0,380)$ & $(-3,271 ;-1,030)$ \\
\hline \multirow{3}{*}{$\beta_{3(\mathrm{GN})}$} & 0,593 & 0,086 & 0,264 & 0,431 \\
& 136 & 65 & 05 & 71 \\
\hline \multirow{3}{*}{$\beta_{4(\mathrm{AN})}$} & $(-0,483 ; 1,716)$ & $(-1,029 ; 1,117)$ & $(-0,800 ; 1,461)$ & $(-0,648 ; 1,424)$ \\
\hline \multirow{3}{*}{$\beta_{5(\mathrm{PKD})}$} & 0,575 & 0,941 & 0,972 & 0,711 \\
& 26 & 20 & 24 & 09 \\
$\lambda_{1}$ & $(-0,599 ; 1,868)$ & $(-0,199 ; 2,193)$ & $(-0,242 ; 2,214)$ & $(-0,465 ; 1,947)$ \\
\hline$\lambda_{2}$ & $-2,801$ & $-2,367$ & $-0,188$ & $-0,555$ \\
& 38 & 16 & 90 & 72 \\
& $0,020 ;-0,770)$ & $(-4,347 ;-0,551)$ & $(-2,937 ; 2,393)$ & $(-3,143 ; 1,825)$ \\
\hline$\lambda_{3}$ & 23 & 0,039 & 0,024 & 0,042 \\
& 0,061 & 91 & 15 & 105 \\
\hline$\lambda_{4}$ & 20 & 0,082 & 0,051 & 0,119 \\
& 0,027 & 61 & 01 & 132 \\
\hline & 0,047 & 0,035 & 0,019 & 0,075 \\
& 56 & 137 & 28 & 408 \\
\hline
\end{tabular}

Baseados nestas análises concluímos que o modelo de regressão final é dado por

$$
S\left(t_{i} ; \boldsymbol{\lambda}, \boldsymbol{\beta}\right)=\exp \left\{-\left[\sum_{k=1}^{j-1} \lambda_{k}\left(s_{k}-s_{k-1}\right)+\lambda_{j}\left(t-s_{j-1}\right)\right] \exp \left\{x_{i 2} \beta_{2}+x_{i 3} \beta_{3}+x_{i 4} \beta_{4}+x_{i 5} \beta_{5}\right\}\right\}
$$


$i=1, \ldots, 38$ e $j=1, \ldots, 4$.

As médias a posteriori, os desvios padrão e a densidade a posteriori máxima (HPD) com $95 \%$ de credibilidade para $\lambda_{j}, j=1, \ldots, 4$ e para os $\beta_{j}$ são apresentados na Tabela 2.7 .

TABELA 2.7: Resumo a posteriori para o modelo final.

\begin{tabular}{cccc}
\hline Parâmetro & Média & Desvio Padrão & HPD $(95 \%)$ \\
\hline$\beta_{2(\text { sexo })}$ & $-1,154$ & 0,493 & $(-2,124 ;-0,186)$ \\
$\beta_{3(\mathrm{GN})}$ & 0,241 & 0,534 & $(-0,743 ; 1,343)$ \\
$\beta_{4(\mathrm{AN})}$ & 0,736 & 0,529 & $(-0,283 ; 1,798)$ \\
$\beta_{5(\mathrm{PKD})}$ & $-2,081$ & 0,956 & $(-4,070 ;-0,339)$ \\
$\lambda_{1}$ & 0,017 & 0,010 & $(0,001 ; 0,038)$ \\
$\lambda_{2}$ & 0,043 & 0,028 & $(0,004 ; 0,098)$ \\
$\lambda_{3}$ & 0,012 & 0,008 & $(0,001 ; 0,029)$ \\
$\lambda_{4}$ & 0,026 & 0,018 & $(0,002 ; 0,060)$ \\
\hline
\end{tabular}

Os valores para os critérios de seleção B e DIC são -159,321 e 315,000 respectivamente. Comparados com os valores da Tabela 2.3 percebemos que temos um melhor ajuste para o modelo. Ainda na Tabela 2.7 podemos notar que as covariáveis sexo e PKD são significativas, ou seja, a infecção renal está relacionada com o sexo do indivíduo em estudo, havendo diferenças entre as taxas de infecção no sexo feminino e masculino e também no tipo de doença, na qual a doença PKD se destaca das outras duas, como podemos observar na Figura 2.5.

Na Figura 2.5 apresentamos as estimativas da função de sobrevivência estratificada por sexo dos indivíduos em estudo de acordo com cada tipo de doença apresentada, podemos notar que a função de sobrevivência de pacientes com doença PKD é superior aos dos pacientes com doenças AN e GN. Também podemos observar que o tempo de sobrevivência é maior em pacientes do sexo feminino. 
(a)

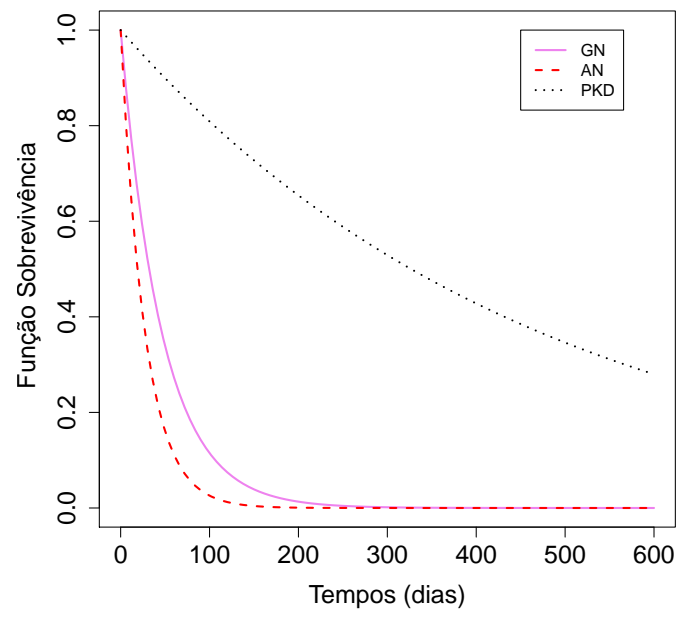

(b)

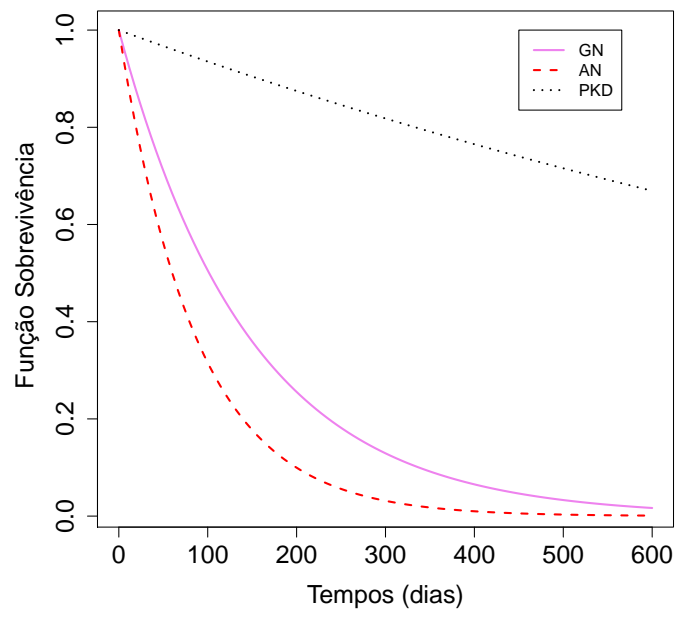

FIGURA 2.5: Estimativas da função de sobrevivência por sexo: (a) sexo masculino e (b) sexo feminino.

\subsection{Conclusões}

Podemos observar por meio dos dados simulados que a divergência de KullbackLeibler é um método eficaz para detectar observações influentes. Pelo conjunto de dados reais identificamos quais pontos influenciaram o ajuste do modelo, pela retirada de tais pontos obtivemos um modelo final e estimamos seus parâmetros. Ainda podemos observar pelos critérios de seleção de modelos que o modelo final obtido tem indícios de ser o ideal para o conjunto de dados considerado. 


\section{Capítulo 3}

\section{Modelo de sobrevivência com fração de}

\section{cura}

Modelos de sobrevivência incorporando fração de cura estão se tornando cada vez mais populares, principalmente na análise dos dados clínicos de câncer, sendo utilizado para modelar dados de tempo do evento para vários tipos de câncer em que uma proporção significativa dos pacientes são "curados". Modelos com fração de cura são baseados em mecanismos de ativação de fatores latentes que envolvem tempos de falha em dois diferentes níveis: um tempo de falha observado $(T)$ que é o tempo observado para ocorrer o evento de interesse e o tempo de um evento latente $\left(R_{i}\right), i=1,2, \ldots M$ que é o tempo de ativação $\operatorname{dos} M$ fatores latentes que geram o tempo de falha observado (T) (Cooner et al., 2007).

\subsection{Formulação do modelo}

Suponha que, para um indivíduo da população, seja denotado por $M$ o número de causas que competem para produzir um evento de interesse. Assumimos que $M$ segue uma determinada distribuição de probabilidade. Além disso, seja denotado por $R_{i}$ o tempo (aleatório) para a $i$-ésima causa produzir o evento de interesse. Isto é, $R_{i}$ pode ser encarada como um tempo de incubação para a $i$-ésima causa. As variáveis $R_{i}, i=1,2, \ldots$, são 
independentes e identicamente distribuídas (i.i.d.) com função de distribuição comum, $F(\cdot)=1-S(\cdot)$ e são independentes de $M$. Assim, o tempo para o evento de interesse ser observado pode ser definido pela variável aleatória

$$
T=\min \left\{R_{1}, \ldots, R_{M}\right\}
$$

$\operatorname{com} P(T=\infty \mid M=0)=1$.

Tsodikov et al. (2003), Rodrigues et al. (2008) entre outros demonstram que $S_{\text {pop }}(t)=\phi(S(t))$, em que $\phi(\cdot)$ é a função geradora de probabilidade para o número de causas competitivas $M$. Desta forma temos que

$$
\begin{aligned}
S_{\text {pop }}(t)= & P(\text { não ocorrer o evento de interesse até o tempo } t) \\
= & P\left(R_{0}>t, M=0\right)+P\left(\min \left\{R_{1}, \ldots, R_{M}\right\}>t, M \geq 1\right) \\
= & P\left(R_{0}>t, M=0\right)+P\left(R_{1}>t, R_{2}>t, \ldots, R_{M}>t, M \geq 1\right) \\
= & P\left(R_{0}>t, M=0\right)+P\left(R_{1}>t, M=1\right)+P\left(R_{1}>t, R_{2}>t, M=2\right)+ \\
& \ldots+P\left(R_{1}>t, \ldots, R_{M}>t, M=m\right)+\ldots \\
= & P\left(R_{0}>t ; M=0\right) P(M=0)+P\left(R_{1}>t ; M=1\right) P(M=1)+ \\
& P\left(R_{1}>t, R_{2}>t, \ldots, R_{M}>t ; M=m\right) P(M=m)+\ldots \\
= & p_{0}+S(t) p_{1}+(S(t))^{2} p_{2}+\ldots+(S(t))^{m} p_{m}+\ldots \\
= & p_{0}+\sum_{m=1}^{\infty} p_{m}(S(t))^{m} \\
= & \phi(S(t))
\end{aligned}
$$

Podemos observar que $S_{\text {pop }}(t)$ não é uma função de sobrevivência própria, isto é $\lim _{t \rightarrow \infty} S_{\text {pop }}(t)>$ 0.

Como em de Castro et al. (2009), consideramos que $M$ segue uma distribuição binomial negativa com parâmetros $\theta$ e $\alpha$ (Piegorsch, 1990), com a seguinte função de 
probabilidade

$$
P(M=m ; \theta, \alpha)=\frac{\Gamma\left(\alpha^{-1}+m\right)}{\Gamma\left(\alpha^{-1}\right) m !}\left(\frac{\theta \alpha}{1+\theta \alpha}\right)^{m}\left(\frac{1}{1+\alpha \theta}\right)^{1 / \alpha}
$$

$m=0,1,2, \ldots, \theta>0$ e $\alpha>-1 / \theta$, de modo que

$$
E[M]=\theta, \quad \operatorname{Var}(M)=\theta+\alpha \theta^{2}
$$

Como observado por Rodrigues et al. (2009a) a variância de $M$ (número de causas ou riscos) em (3.3) proporciona ao mesmo tempo dois cenários importantes, o de sobredispersão que ocorre quando $\alpha>0$ e pode ser interpretado no caso de pesquisas biológicas na área de câncer como um agrupamento de células cancerígenas ou tumorais e a subdispersão que ocorre no sentido inverso quando $\alpha$ assume valores negativos. A distribuição de probabilidade em (3.2) é muito flexível no sentido de fornecer ligações entre as distribuições geométrica, Poisson e binomial negativa, pois se $\alpha=1$ temos o modelo geométrico e se $\alpha \rightarrow 0$ temos o modelo de Poisson.

A função geradora de probabilidade para o modelo binomial negativo é dada por

$$
\phi(s)=\left[\frac{1}{1+\alpha \theta(1-s)}\right]^{1 / \alpha}, 0 \leq s \leq 1
$$

De (3.1) e (3.4) a função de sobrevivência populacional é expressa por

$$
S_{\text {pop }}(t)=[1+\alpha \theta F(t)]^{-1 / \alpha},
$$

A proporção de indivíduos curados é determinada por $p_{0}=\lim _{t \rightarrow \infty} S_{\text {pop }}(t)$, neste caso temos $p_{0}=[1+\alpha \theta]^{\frac{-1}{\alpha}}$. Da equação (3.5) podemos obter a função densidade populacional

$$
\begin{aligned}
f_{\mathrm{pop}}(t) & =-\frac{d}{d t}\left(S_{\mathrm{pop}}(t)\right) \\
& =\theta f(t)[1+\alpha \theta F(t)]^{-(1+1 / \alpha)}
\end{aligned}
$$


em que $f(t)=\frac{d}{d t}(F(t))$.

\subsection{Função de Verossimilhança}

Supomos $m$ indivíduos e $M_{i}$ a variável que representa o número de causas competindo que podem produzir um câncer detectável para o $i$-ésimo indivíduo. Assumimos que os $M_{i}$ 's são variáveis aleatórias com distribuição binomiais negativas i.i.d. com média $\theta_{i}, i=1, \ldots, m$. Nós enfatizamos aqui que os $M_{i}$ 's não são observados e podem ser vistos como variáveis latentes. Entretanto, supomos que $R_{i 1}, \ldots, R_{i M_{i}}$ são os tempos de incubação i.i.d. para cada célula cancerígena $M_{i}$ do $i$-ésimo indivíduo, $i=1, \ldots, m$ e todos tem função de distribuição acumulada $F(\cdot \mid \boldsymbol{\lambda})$. Seja $Y_{i}$ o tempo observado tal que, $Y_{i}=\min \left(T_{i}, C_{i}\right), \operatorname{com} T_{i}=\min \left\{R_{i 1}, \ldots, R_{i M_{i}}\right\}, C_{i}$ o tempo de censura e $\delta_{i}$ o indicador de censura igual a 1 se $Y_{i}=T_{i}$ e 0 caso contrário. Nós incluímos as covariáveis no modelo por meio do valor esperado do número de causas que competem pra produzir um câncer, ou seja, $E\left(M_{i}\right)=\theta_{i}=\exp \left(\boldsymbol{x}_{\boldsymbol{i}}^{\top} \boldsymbol{\beta}\right), i=1, \ldots, m$, em que $\boldsymbol{\beta}$ é um vetor $k \times 1$ de coeficientes de regressão. Os dados observados são $\mathcal{D}=(n, \boldsymbol{y}, \boldsymbol{\delta}, \boldsymbol{X})$, com $\boldsymbol{y}=\left(y_{1}, \ldots, y_{m}\right)^{\top}, \boldsymbol{\delta}=\left(\delta_{1}, \ldots, \delta_{m}\right)^{\top}$ e $\boldsymbol{X}$ uma matrix $m \times k$ que contém as covariáveis.

Baseados na distribuição binomial negativa em (3.2) com $\alpha>0$ e, considerando o modelo exponencial por partes para os tempos de promoção $R_{i}$ como apresentado na Seção $2.1, \mathrm{com}$

$$
f_{j}\left(y_{i} ; \boldsymbol{\lambda}\right)=\lambda_{j} \exp \left\{-\left[\sum_{k=1}^{j-1} \lambda_{k}\left(s_{k}-s_{k-1}\right)+\lambda_{j}\left(y_{i}-s_{j-1}\right)\right]\right\}
$$

e

$$
F_{j}\left(y_{i} ; \boldsymbol{\lambda}\right)=1-\exp \left\{-\left[\sum_{k=1}^{j-1} \lambda_{k}\left(s_{k}-s_{k-1}\right)+\lambda_{j}\left(y_{i}-s_{j-1}\right)\right]\right\},
$$

podemos expressar a função de verossimilhança da seguinte forma

$$
L(\boldsymbol{\gamma} ; \mathcal{D}) \propto \prod_{i=1}^{m} \prod_{j=1}^{J}\left\{\exp \left(\boldsymbol{x}_{\boldsymbol{i}}^{\top} \boldsymbol{\beta}\right) f_{j}\left(y_{i} ; \boldsymbol{\lambda}\right)\right\}^{\delta_{i} \nu_{i j}}\left\{1+\alpha \exp \left(\boldsymbol{x}_{\boldsymbol{i}}^{\top} \boldsymbol{\beta}\right) F_{j}\left(y_{i} ; \boldsymbol{\lambda}\right)\right\}^{-\nu_{i j}\left(\delta_{i}+1 / \alpha\right)}
$$

em que, $\boldsymbol{\gamma}=(\boldsymbol{\lambda}, \alpha, \boldsymbol{\beta})$ é o vetor de parâmetros, $\mathcal{D}=(n, \boldsymbol{y}, \boldsymbol{\delta}, \boldsymbol{X}, \boldsymbol{\nu}), \boldsymbol{\nu}=\left(\nu_{11}, \ldots, \nu_{m J}\right)$ 
com $\nu_{i j}=1$ se $s_{j-1}<y_{i} \leq s_{j}$ e $\nu_{i j}=0$ caso contrário, $j=1, \ldots, J$ e $i=1, \ldots, m$.

\subsection{Inferência bayesiana}

Assumindo independência entre os parâmetros do modelo a densidade a priori conjunta é expressa por

$$
\pi(\alpha, \boldsymbol{\beta}, \boldsymbol{\lambda})=\pi(\alpha) \pi(\boldsymbol{\beta}) \pi(\boldsymbol{\lambda})
$$

em que o parâmetro $\alpha$ tem distribuição $G(a, b)$, com $G(a, b)$ denotando uma distribuição gama com média $a / b$ e variância $a / b^{2} . \boldsymbol{\beta}_{\boldsymbol{l}} \sim \pi\left(\boldsymbol{\beta}_{l}\right), l=1, \ldots, k$ e $\boldsymbol{\lambda}_{j} \sim \pi\left(\boldsymbol{\lambda}_{j}\right), j=1, \ldots, J$. Combinando essa densidade a priori com a verossimilhança (3.9), a densidade a posteriori conjunta é dada por

$$
\pi(\alpha, \boldsymbol{\beta}, \boldsymbol{\lambda} \mid \mathcal{D})=L(\boldsymbol{\gamma} ; \mathcal{D}) \pi(\alpha) \pi(\boldsymbol{\beta}) \pi(\boldsymbol{\lambda})
$$

Como a densidade a posteriori conjunta não é uma densidade padrão usamos métodos de Monte Carlo via cadeias de Markov (MCMC), tais como o amostrador de Gibbs e Metropolis-Hasting. Podemos mostrar que as densidades a posteriori condicionais para o amostrador de Gibbs são expressas por

$$
\begin{aligned}
& \pi(\boldsymbol{\beta} \mid \alpha, \boldsymbol{\lambda}, \mathcal{D}) \propto L(\boldsymbol{\gamma} ; \mathcal{D}) \pi(\boldsymbol{\beta}) \\
& \pi(\boldsymbol{\lambda} \mid \boldsymbol{\beta}, \boldsymbol{\alpha}, \mathcal{D}) \propto L(\boldsymbol{\gamma} ; \mathcal{D}) \pi(\boldsymbol{\lambda}) \\
& \pi(\alpha \mid \boldsymbol{\beta}, \boldsymbol{\lambda}, \mathcal{D}) \propto L(\boldsymbol{\gamma} ; \mathcal{D}) \pi(\alpha)
\end{aligned}
$$

Como as densidades a posteriori condicionais não possuem forma fechada usaremos o algoritmo de Metropolis-Hastings dentro do ciclo do algoritmo de Gibbs (Gilks et al., 1996) para gerar amostras dos parâmetros envolvidos no modelo. 


\subsection{Aplicação}

Nesta seção ilustraremos a metodologia descrita anteriormente com um conjunto de dados simulados e um conjunto de dados reais.

\subsubsection{Dados simulados}

Para examinar o desempenho das medidas de diagnósticos propostas neste trabalho, nós consideramos um conjunto de dados simulados, em que a covariável $x_{i}, i=1, \ldots n$ foi gerada de uma distribuição normal $(1 ; 0,5)$. Os tempos de falha $T_{i}$ para o modelo (3.1) foram simulados de uma distribuição exponencial com parâmetro $\lambda=\exp (-3)$, consideramos a proporção de curados $\left.p_{0 i}=\left[1+\alpha \theta_{i}\right)\right]^{-1 / \alpha} \operatorname{com} \alpha=2, \theta_{i}=\exp \left(\beta_{0}+\beta_{1} x_{i}\right)$ com $\beta_{0}=-0,5$ e $\beta_{1}=0,7$ e a proporção de observações censuradas $p_{c i}=p_{0 i}+0,07$. Os tempos observados e os indicadores de censura foram gerados como segue:

1. Gerar $u_{i} \sim$ uniforme $(0,1)$;

2. Se $u_{i}<p_{0 i}$, fazer $t_{i}=\infty$; caso contrário, fazer

$$
t_{i}=F^{-1}\left(\frac{u_{i}^{-\alpha}-1}{\alpha \exp \left(\beta_{0}+\beta_{1} x_{i}\right)}\right)
$$

$\operatorname{com} F^{-1}(t)=-\log (1-t) / e^{\lambda}$

3. Gerar

$$
c_{i} \sim \exp \left(\frac{e^{\alpha}\left(p_{c i}-p_{0 i}\right)}{1-\left(p_{c i}-p_{0 i}\right)}\right)
$$

4. Considerar $y_{i}=\min \left\{t_{i}, c_{i}\right\}$;

5. Se $y_{i}<c_{i}$, então $\delta_{i}=1$, caso contrário, temos $\delta_{i}=0, i=1, \ldots, n$.

Para a análise bayesiana consideramos densidades a priori independentes para os parâmetros do modelo, com $\beta_{l} \sim N(0 ; 100)$, com $l=0,1 ; \alpha \sim G(1 ; 0,01)$ e $\lambda_{j} \sim$ $G(1 ; 0,001)$, com $j=1$. Considerando essas densidades a priori geramos amostras de Gibbs 
através do algoritmo de Gibbs com Metropolis-Hasting da seguinte forma: geramos duas cadeias paralelas cada uma com 35.000 iterações para cada parâmetro, descartamos as primeiras 5.000 iterações afim de eliminar os efeitos dos valores iniciais e evitar problemas de correlação, consideramos espaçamento de tamanho 10, resultando uma amostra de Gibbs de tamanho 6.000. Para monitorar a convergência do amostrador de Gibbs utilizamos a aproximação desenvolvida por Gelman e Rubin(1992). Afim de avaliar a robustez do modelo relacionado às escolhas dos hiperparâmetros das priori, um estudo de sensibilidade foi realizado, no qual constatamos que as estimativas dos parâmetros a posteriori não apresentaram diferenças significativas.

Selecionamos os casos 14, 54 e 80 para perturbar afim de criarmos observações influentes no conjunto de dados. Nós escolhemos um ou dois casos e perturbamos a variável resposta da seguinte maneira $\tilde{t}_{i}=t_{i}+6 \tau_{t}$, em que $\tau_{t}$ são os desvios padrão dos tempos $t_{i}$. Na Tabela 3.1 apresentamos as estimativas a posteriori para os dados simulados. O conjunto de dados $(a)$ denota os dados originais simulados sem nenhuma perturbação e os conjuntos de dados $(b)-(f)$ denota os dados com casos perturbados, informamos ainda na Tabela 3.2 os valores para os critérios de seleção $B$ e $D I C$ (discutidos na seção 1.1). Esses critérios demonstram que o modelo sem casos perturbados $(a)$ apresenta o melhor ajuste para os dados.

TABELA 3.1: Médias e desvios padrão a posteriori para os parâmetros do modelo dos dados simulados com fração de cura.

\begin{tabular}{cccccccccc}
\hline Dados & Casos & \multicolumn{2}{c}{$\alpha$} & \multicolumn{2}{c}{$\beta_{0}$} & \multicolumn{2}{c}{$\beta_{1}$} & \multicolumn{2}{c}{$\lambda_{1}$} \\
& perturbados & Média & DP & Média & DP & Média & DP & Média & DP \\
\hline a & Nenhum & 1,6764 & 1,2652 & $-0,5200$ & 0,6972 & 0,5397 & 0,4917 & 0,0423 & 0,0127 \\
b & 14 & 6,3882 & 1,4722 & 3,0854 & 1,3681 & 1,0965 & 0,8882 & 0,0011 & 0,0009 \\
c & 54 & 6,7836 & 1,4893 & 3,8010 & 1,4194 & 0,6344 & 0,9039 & 0,0010 & 0,0008 \\
d & 80 & 6,5851 & 1,3550 & 4,5487 & 1,6104 & $-0,0673$ & 0,8929 & 0,0010 & 0,0008 \\
e & $\{14,54\}$ & 6,4562 & 1,3063 & 4,5235 & 1,5415 & 0,5336 & 0,8627 & 0,0004 & 0,0004 \\
f & $\{14,54,80\}$ & 6,7219 & 1,3998 & 4,8643 & 1,3707 & 0,1222 & 0,8458 & 0,0005 & 0,0004 \\
\hline
\end{tabular}

TABELA 3.2: Critérios de seleção de modelos.

\begin{tabular}{ccccccc}
\hline Critério & \multicolumn{5}{c}{ Dados } \\
& $\mathrm{a}$ & $\mathrm{b}$ & $\mathrm{c}$ & $\mathrm{d}$ & $\mathrm{e}$ & $\mathrm{f}$ \\
\hline B & $-229,1443$ & $-247,1962$ & $-245,8269$ & $-247,5562$ & $-253,4850$ & $-251,3234$ \\
DIC & 458,1000 & 489,3000 & 485,2000 & 490,1000 & 505,0000 & 501,3000 \\
\hline
\end{tabular}


Considerando as amostras de Gibbs, foram estimadas as medidas de divergência de K-L (apresentado na Seção 1.2.1) para cada um dos casos (a,b,c,d,e e $f$ ), esses resultados são graficados na Figura 3.1. Ainda foram estimadas as medidas de calibração (discutidos na Seção 1.2.1) referentes a cada caso. Os resultados apresentados na Tabela 3.3 indicam que sem perturbação nos dados $(a)$, os casos selecionados não são influentes, pois obtivemos valores pequenos para $K\left(P, P_{(-i)}\right)$ em cada caso. Entretanto, após perturbação $(b-f)$ obtivemos medidas maiores de $K\left(P, P_{(-i)}\right)$ e medidas de calibração maiores que 0,5 e próximas ao valor 1 indicando que estes casos são influentes.

TABELA 3.3: Medidas de diagnósticos para os dados simulados com fração de cura .

\begin{tabular}{cccc}
\hline Nomes dos Dados & Identificação dos casos & $K\left(P, P_{(-i)}\right)$ & Calibração \\
\hline a & 14 & 0,0817 & 0,6942 \\
& 54 & 0,0265 & 0,6136 \\
b & 80 & 0,1159 & 0,7274 \\
c & 14 & 2,1781 & 0,9967 \\
d & 54 & 2,7368 & 0,9989 \\
e & 80 & 1,9271 & 0,9946 \\
& 14 & 0,3117 & 0,8405 \\
& 80 & 0,2130 & 0,7945 \\
f & 54 & 0,3280 & 0,8468 \\
& 80 & 0,2108 & 0,7932 \\
\hline
\end{tabular}

Na Figura 3.1 plotamos $K\left(P, P_{(-i)}\right)$ para o modelo proposto, podemos notar que K-L identifica os casos influentes apresentando valores maiores de $K\left(P, P_{(-i)}\right)$ para os casos perturbados se comparados aos outros casos. 
(a)

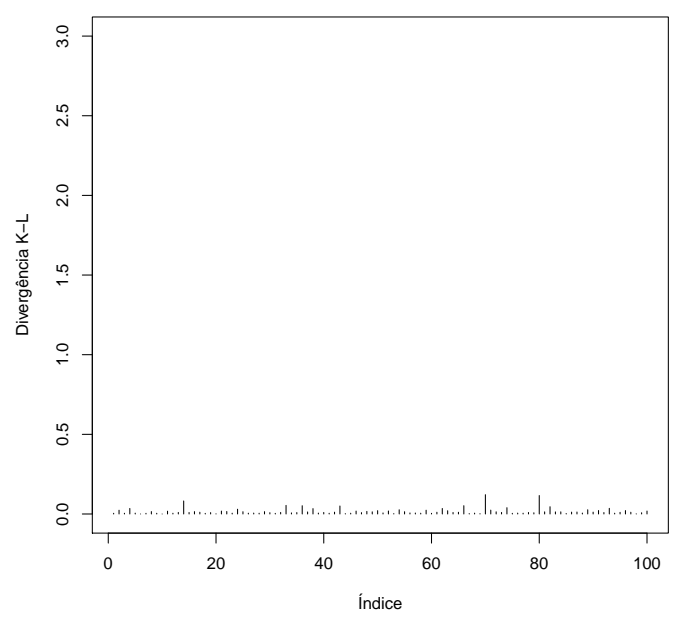

(c)

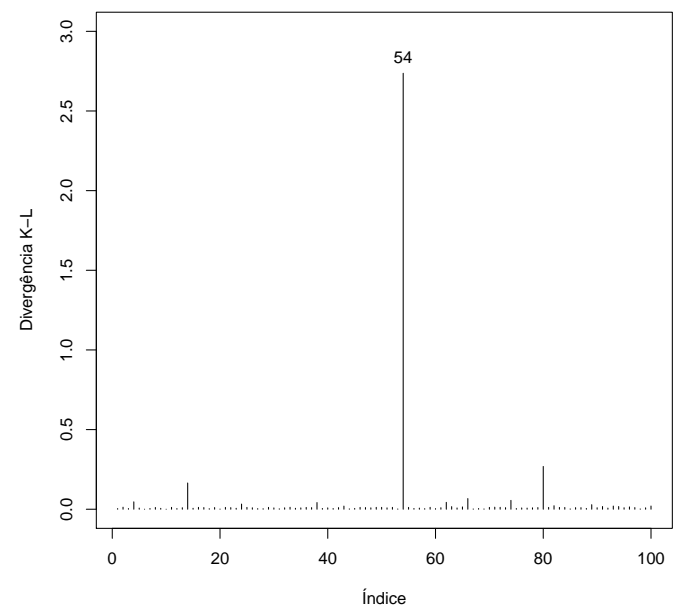

(e)

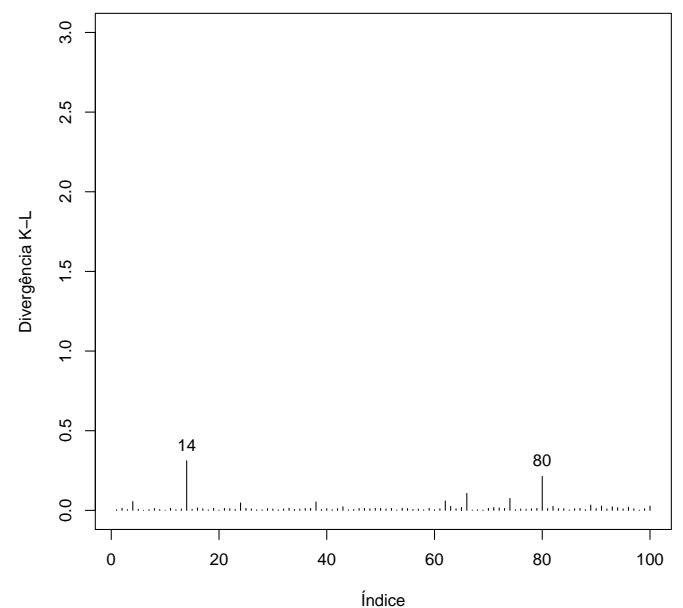

(b)

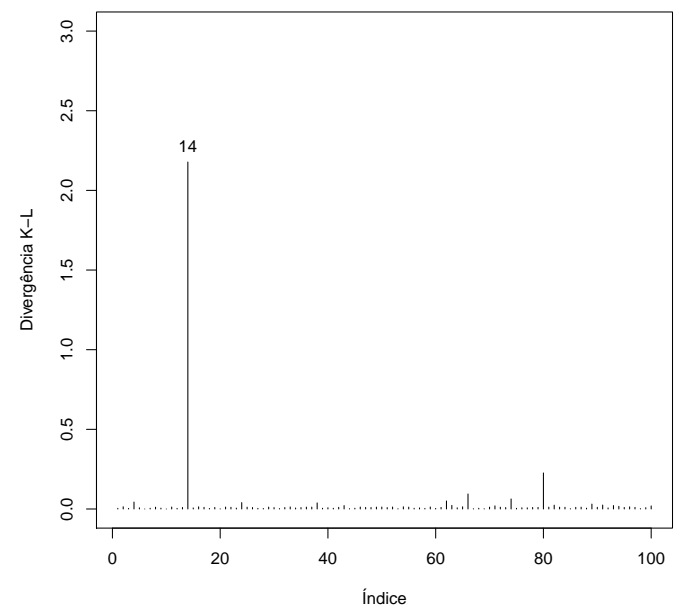

(d)

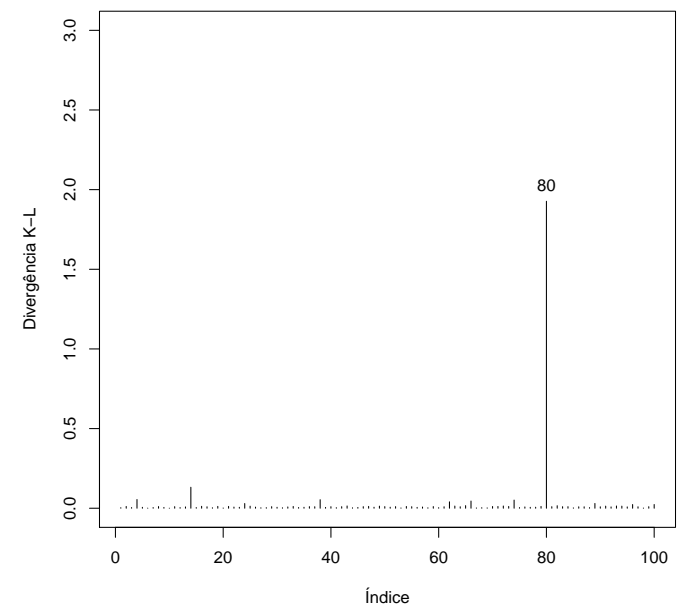

(f)

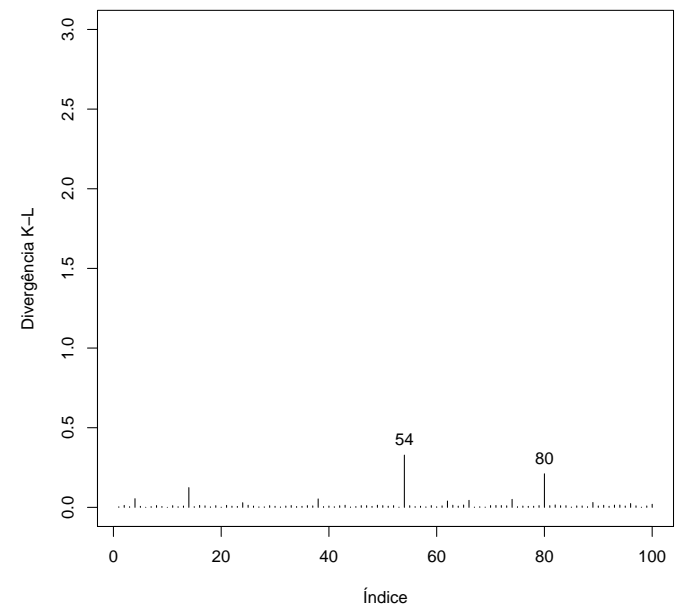

FIGURA 3.1: Gráfico de índices de $K\left(P, P_{(-i)}\right)$ para os dados simulados com fração de cura. 


\subsubsection{Dados de melanoma}

Os dados considerados nesta seção provém de um estudo de melanoma com o objetivo de avaliar a eficácia da aplicação de uma dosagem alta de interferon alfa-2b como forma de prevenir a recorrência de câncer. Os dados foram obtidos entre 1991 e 1995, mas houve acompanhamento dos pacientes até 1998. A variável resposta (y) representa o tempo até a morte dos paciente ou tempo de censura. Maiores detalhes deste conjunto de dados pode ser encontrado em Ibrahim et al. (2001). Da amostra original foram desconsiderados 10 pacientes devido a presença de dados faltantes, resultando em $n=417$ pacientes, com $56 \%$ de observações censuradas. As variáveis consideradas neste estudo incluem $y$ : tempo (em anos); $x_{1}$ : categoria do nódulo $(1,2,3,4) ; x_{2}$ : idade (anos); $x_{3}$ : espessura do tumor(em $\mathrm{mm})$.

Na Figura 3.2 apresentamos a estimativa de Kaplan-Meier da função de sobrevivência por categoria do nódulo para os dados de melanoma, na qual observamos a existência de uma apreciável fração de indivíduos "curados", pelo menos no que diz respeito ao intervalo de tempo abrangido pelo estudo.

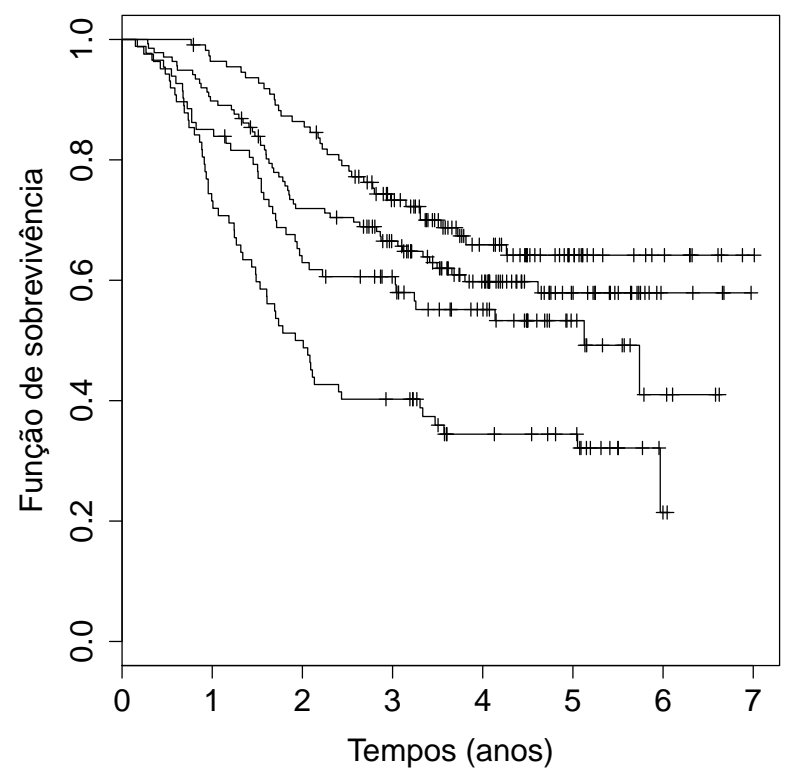

FIGURA 3.2: Estimativa de Kaplan-Meier da função de sobrevivência por categoria nódulo (1 a 4 , de cima para baixo). 
Uma análise bayesiana foi considerada e para cada componente de $\boldsymbol{\beta}$, consideramos densidades a priori independentes com uma distribuição normal com média 0 e variância 1000. Para $\lambda_{1}, \ldots, \lambda_{J}$, consideramos uma distribuição gama, ou seja, $\lambda_{j} \sim G(1 ; 0.01)$, $j=1, \ldots$ J. Para o parâmetro $\alpha$ consideramos uma distribuição a priori informativa, $\alpha \sim$ $G(1 ; 1)$ com a finalidade de garantir que a distribuição a posteriori seja própria (Chen et al., 1999). Assim como no Capítulo 2 temos que a distribuição a posteriori para os parâmetros obtida não é tratável analiticamente, portanto métodos MCMC podem ser considerados. Como as condicionais não tem forma fechada geramos amostras das distribuição a posteriori dos parâmetros usando o algoritmos de Gibbs com Metropolis-Hasting da seguinte forma: geramos duas cadeias paralelas cada uma com 35.000 iterações com um burn in de 5.000 e saltos de tamanho 10, resultando uma amostra de Gibbs de tamanho 6.000. Para monitorar a convergência do amostrador de Gibbs utilizamos a aproximação desenvolvida por Gelman e Rubin(1992). Afim de avaliar a robustez do modelo relacionado às escolhas dos hiperparâmetros das priori, um estudo de sensibilidade foi realizado, no qual constatamos que as estimativas dos parâmetros a posteriori não apresentaram diferenças significativas. A Tabela 3.4 apresenta os valores para os critérios de seleção $B$ e $D I C$ para os modelos ajustados. Ambos critérios indicam $J=5$ como melhor partição do eixo dos tempos.

TABELA 3.4: Critérios de seleção de modelos de acordo com cada partição.

\begin{tabular}{ccc}
\hline Modelo & \multicolumn{2}{c}{ Critério } \\
$\mathrm{J}$ & $B$ & $D I C$ \\
\hline 1 & $-536,5428$ & 1068 \\
2 & $-533,1140$ & 1066 \\
3 & $-525,3784$ & 1051 \\
4 & $-524,8778$ & 1050 \\
5 & $-517,3055$ & 1035 \\
6 & $-521,7236$ & 1043 \\
\hline
\end{tabular}

Na Tabela 3.5 apresentamos o resumo a posteriori dos parâmetros do modelo com o melhor ajuste dentre os considerados. Para todos os parâmetros observamos valores de $\hat{R}$ próximos a 1 , indicando que as iterações foram suficientes para se chegar a convergência. A " highest posterior density" (HPD)(Chen \& Shao, 1999) com 90\% de credibilidade indica que somente a covariável, que representa a espessura do tumor é não significativa. 
TABELA 3.5: Resumo a posteriori dos parâmetros do modelo.

\begin{tabular}{ccccc}
\hline Parâmetro & Média & Desvio Padrão & $H P D(90 \%)$ & $\hat{R}$ \\
\hline$\alpha$ & 1,1628 & 0,6455 & $(0,0305 ; 2,0301)$ & 1,0181 \\
$\beta_{0}$ (intercepto) & $-1,9439$ & 0,5011 & $(-2,7961 ;-1,1754)$ & 1,0044 \\
$\beta_{1}$ (cat. nódulo) & 0,5500 & 0,1320 & $(0,3326 ; 0,7601)$ & 1,0091 \\
$\beta_{2}$ (idade) & 0,0143 & 0,0076 & $(0,0021 ; 0,0271)$ & 1,0068 \\
$\beta_{3}$ (espessura) & 0,0326 & 0,0308 & $(-0,0208 ; 0,0799)$ & 1,0022 \\
$\lambda_{1}$ & 0,0869 & 0,0314 & $(0,0350 ; 0,1350)$ & 1,0238 \\
$\lambda_{2}$ & 0,2166 & 0,0663 & $(0,1038 ; 0,3183)$ & 1,0045 \\
$\lambda_{3}$ & 0,5312 & 0,1513 & $(0,2837 ; 0,7777)$ & 1,0218 \\
$\lambda_{4}$ & 0,4280 & 0,1280 & $(0,2257 ; 0,6366)$ & 1,0044 \\
$\lambda_{5}$ & 0,4255 & 0,1630 & $(0,1631 ; 0,6811)$ & 1,0030 \\
\hline
\end{tabular}

Com as amostras de Gibbs foram estimadas as medidas de divergência de $\mathrm{K}-\mathrm{L}$ para cada uma das observações, esses resultados podem ser observados na Figura 3.3, em que destacamos as observações 23, 47, 176 e 330 que apresentaram maiores valores quando comparados com as demais observações. Para verificar se essas observações são influentes estimamos a calibração da medida de divergência $\mathrm{K}-\mathrm{L}$, essas estimativas são apresentados na Tabela 3.6 conjuntamente com as respectivas estimativas da divergência $\mathrm{K}-$ L $\left(K\left(P, P_{-i}\right)\right)$, podemos observar pelos valores da calibração que nenhuma das observações são influentes, pois deveríamos ter resultados similares aos apresentados na Tabela 3.3.

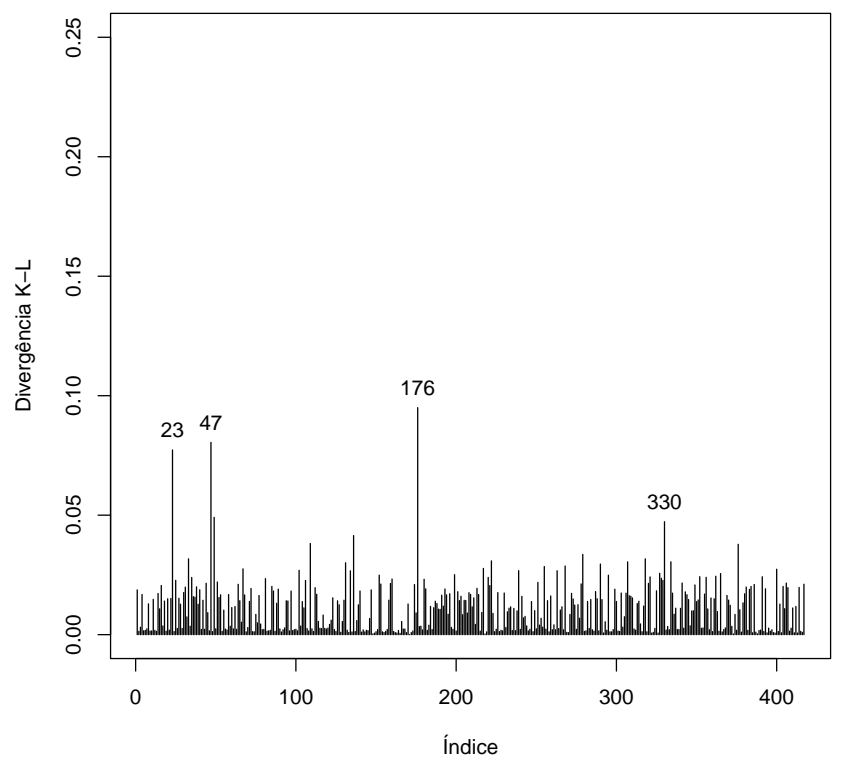

FIGURA 3.3: Gráfico de índices de $K\left(P, P_{-i}\right)$ para dados de melanoma. 
A título de ilustração realizamos uma análise de sensibilidade na estimativa dos parâmetros a posteriori, considerando as três observações com maiores valores de calibração.

TABELA 3.6: Identificação dos casos influentes para dados de melanoma.

\begin{tabular}{ccc}
\hline Identificação do caso & \multicolumn{2}{c}{ Influência caso a caso } \\
\hline Paciente & $K\left(P, P_{-i}\right)$ & Calibração \\
\hline 23 & 0,0773 & 0,6892 \\
47 & 0,0804 & 0,6927 \\
176 & 0,0950 & 0,7079 \\
330 & 0,0472 & 0,6501 \\
\hline
\end{tabular}

Na Tabela 3.7 apresentamos as novas estimativas dos parâmetros após a exclusão das observações "influentes", uma a uma ou excluindo todas de uma vez, representado por $I=\{23 ; 47 ; 176\}$, também calculamos as variações relativas $(V R)$ (em porcentagem), as quais são obtidas por $V R_{\gamma_{d}}=\left|\left(\hat{\gamma}_{d}-\hat{\gamma}_{d(I)}\right) / \hat{\gamma}_{d}\right| \times 100 \%$, em que $\hat{\gamma}_{d(I)}$ denota a média a posteriori de $\gamma_{d}$, com $d=1, \ldots, 10$ após o conjunto $I$ de observações ter sido removido. A densidade a posteriori máxima (HPD) com $90 \%$ de credibilidade para cada nova estimativa é apresentada entre parênteses na Tabela 3.7. 
TABELA 3.7: Estimativas, $V R$ (em \%) e a correspondente HPD (90\%) ajustados para o conjunto de dados de melanoma.

\begin{tabular}{ccccc}
\hline Parâmetros & \multicolumn{4}{c}{ Observações descartadas } \\
& 23 & 47 & 176 & $I$ \\
\hline \multirow{2}{*}{$\alpha$} & 1,1257 & 1,1321 & 1,1943 & 0,9802 \\
& 3,1 & 2,6 & 2,7 & 15,7 \\
& $(0,0482 ; 2,0205)$ & $(0,0709 ; 2,0923)$ & $(0,1309 ; 2,1762)$ & $(0,0002 ; 1,8020)$ \\
\hline \multirow{3}{*}{$\beta_{0}$ (intercepto) } & $-1,9694$ & $-1,9649$ & $-1,9100$ & $-1,8940$ \\
& 1,3 & 1,0 & 1,7 & 2,5 \\
& $(-2,7262 ;-1,1558)$ & $(-2,8593 ;-1,0755)$ & $(-2,9123 ;-0,9971)$ & $(-2,7013 ;-1,0281)$ \\
\hline \multirow{3}{*}{$\beta_{1}$ (cat. nódulo) } & 0,5434 & 0,5567 & 0,5436 & 0,5094 \\
& 1,2 & 1,2 & 1,1 & 7,3 \\
& $(0,3373 ; 0,7368)$ & $(0,3342 ; 0,7571)$ & $(0,3490 ; 0,7381)$ & $(0,3102 ; 0,7115)$ \\
\hline \multirow{3}{*}{$\beta_{2}$ (idade) } & 0,0137 & 0,0139 & 0,0133 & 0,0127 \\
& 4,1 & 2,7 & 6,9 & 11,1 \\
\hline \multirow{3}{*}{$\beta_{3}($ espessura $)$} & $0,0015 ; 0,0255)$ & $(-0,0004 ; 0,0264)$ & $(-0,0011 ; 0,0262)$ & $(-0,0002 ; 0,0253)$ \\
\hline$\lambda_{1}$ & 0,0306 & 0,0413 & 0,0323 & 0,0307 \\
& 6,1 & 26,6 & 0,9 & 5,8 \\
\hline$\lambda_{2}$ & $(-0,0179 ; 0,0792)$ & $(-0,0128 ; 0,0868)$ & $(-0,0205 ; 0,0826)$ & $(-0,0139 ; 0,0811)$ \\
\hline$\lambda_{3}$ & 0,0992 & 0,0869 & 0,0939 & 0,1053 \\
& 14,1 & 0,0 & 8,0 & 21,1 \\
\hline$\lambda_{4}$ & 0,2264 & 0,2184 & 0,2180 & 0,2436 \\
& 4,5 & 0,8 & 0,6 & 12,4 \\
\hline$\lambda_{5}$ & 0,5560 & 0,5212 & 0,5307 & 0,5856 \\
& 4,6 & 1,8 & 0,0 & 10,2 \\
\hline Critério $B$ & 0,4737 & 0,4108 & 0,4526 & 0,4839 \\
\hline & 10,6 & 4,0 & 5,7 & 13,0 \\
\hline & 0,4954 & 0,4059 & 0,4695 & 0,5042 \\
& 16,4 & 4,6 & 10,3 & 18,4 \\
\hline & $-516,6873$ & $-516,7518$ & $-516,3218$ & $-516,1163$ \\
\hline
\end{tabular}

Notamos maiores valores de $V R$ para o parâmetro $\widehat{\beta}_{3}$ quando há exclusão da observação 47, o mesmo ocorre com o parâmetro $\widehat{\alpha}$ quando excluímos as observações do conjunto $I$, de maneira geral temos as maiores variações relativas $(V R)$ nos parâmetros $\widehat{\lambda}$ quando excluímos o conjunto $I$. O parâmetro $\widehat{\beta}_{2}$ apresenta sensibilidade em relação as observações "influentes", pois quando excluímos as observações 47 ou 176 há alteração na significância do parâmetro, resumidamente, $\widehat{\beta}_{2}$ passa a ser não significativo no modelo. Observamos que a exclusão de qualquer observação praticamente não altera o valor do critério de selção $B$.

De acordo com o exposto, sugerimos como alternativa a um modelo final excluir apenas a covariável $x_{3}$ que é não significativa. Um resumo a posteriori dos parâmetros do modelo final é apresentado na Tabela 3.8. 
TABELA 3.8: Resumo dos parâmetros a posteriori para o modelo final.

\begin{tabular}{cccc}
\hline Parâmetro & Média & Desvio Padrão & HPD $(90 \%)$ \\
\hline$\alpha$ & 1,1680 & 0,6801 & $(0,0107 ; 2,0978)$ \\
$\beta_{0 \text { (intercepto) }}$ & $-1,7930$ & 0,4672 & $(-2,5175 ;-0,9859)$ \\
$\beta_{1 \text { (cat. nódulo) }}$ & 0,5242 & 0,1212 & $(0,3320 ; 0,7245)$ \\
$\beta_{2 \text { (idade) }}$ & 0,0150 & 0,0077 & $(0,0023 ; 0,0275)$ \\
$\lambda_{1}$ & 0,0878 & 0,0324 & $(0,0339 ; 0,1382)$ \\
$\lambda_{2}$ & 0,2176 & 0,0655 & $(0,1060 ; 0,3185)$ \\
$\lambda_{3}$ & 0,5267 & 0,1494 & $(0,2887 ; 0,7707)$ \\
$\lambda_{4}$ & 0,4268 & 0,1247 & $(0,2231 ; 0,6190)$ \\
$\lambda_{5}$ & 0,4291 & 0,1585 & $(0,1875 ; 0,6896)$ \\
\hline
\end{tabular}

Foram obtidos os valores dos critérios de seleção, $B=-516,8008$ e $D I C=1033$, que comparados aos valores da Tabela 3.4 nos leva a atestar que este modelo é o modelo com melhor ajuste aos dados. Comparando os valores da Tabela 3.8 aos da Tabela 3.5 percebemos que maiores alterações nos resumos a posteriori dos parâmetros do modelo estão relacionados aos coeficientes $\boldsymbol{\beta}$.

Na Tabela 3.9 apresentamos as estimativas da proporção de curados por categoria de nódulo para o modelo final, as quais revelam que quanto maior a categoria do nódulo menor a proporção de curados.

Na Tabela 3.10 avaliamos a proporção de indivíduos curados em cada categoria de nódulo de acordo com a variação de suas idades. Consideramos os quantis 5\%, 50\% e 95\% das idades que correspondem a 29, 47 e 70 anos respectivamente, notamos que com o aumento da idade a proporção de indivíduos curados diminui em cada categoria de nódulo. Podemos concluir que as covariáveis idade e categoria do nódulo influenciam na proporção de indivíduos curados.

TABELA 3.9: Resumo a posteriori para a fração de cura estratificada por categoria do nódulo.

\begin{tabular}{ccccc}
\hline Fração Cura & & & \multicolumn{2}{c}{ Percentil } \\
& Média & Desvio padrão & $2,5 \%$ & $97,5 \%$ \\
\hline$p_{0_{1}}$ & 0,6459 & 0,0490 & 0,5367 & 0,7273 \\
$p_{0_{2}}$ & 0,5267 & 0,0448 & 0,4249 & 0,5989 \\
$p_{0_{3}}$ & 0,4046 & 0,0449 & 0,3039 & 0,4793 \\
$p_{0_{4}}$ & 0,2940 & 0,0499 & 0,1860 & 0,3861 \\
\hline
\end{tabular}


TABELA 3.10: Resumo a posteriori para a fração de cura $\left(p_{0}\right)$ estratificada por categoria do nódulo e por idade.

\begin{tabular}{cccccc}
\hline \multirow{2}{*}{ Idade } & Fração de Cura & Média & Desvio Padrão & $2,50 \%$ & $97,50 \%$ \\
\hline \multirow{2}{*}{29} & $p_{0_{1}}$ & 0,7016 & 0,0543 & 0,5822 & 0,7935 \\
& $p_{0_{2}}$ & 0,5892 & 0,0549 & 0,4724 & 0,6849 \\
& $p_{0_{3}}$ & 0,4672 & 0,0563 & 0,3507 & 0,5664 \\
47 & $p_{0_{4}}$ & 0,3498 & 0,0595 & 0,2256 & 0,4632 \\
& $p_{0_{1}}$ & 0,6459 & 0,0490 & 0,5367 & 0,7273 \\
& $p_{0_{2}}$ & 0,5267 & 0,0448 & 0,4249 & 0,5989 \\
70 & $p_{0_{3}}$ & 0,4046 & 0,0449 & 0,3039 & 0,4793 \\
& $p_{0_{4}}$ & 0,2940 & 0,0499 & 0,1860 & 0,3861 \\
& $p_{0_{1}}$ & 0,5679 & 0,0623 & 0,4384 & 0,6802 \\
& $p_{0_{2}}$ & 0,4459 & 0,0576 & 0,3280 & 0,5532 \\
& $p_{0_{3}}$ & 0,3302 & 0,0554 & 0,2168 & 0,4338 \\
& $p_{0_{4}}$ & 0,2326 & 0,0556 & 0,1191 & 0,3407 \\
\hline
\end{tabular}

A Figura 3.4 ilustra os resultados mostrados na Tabela 3.10. É fácil notar que com o aumento da idade dos pacientes e quanto maior a categoria do nódulo, menor a fração de cura. Há uma diferença significativa na proporção de curados entre a primeira categoria de nódulo e a última. Já entre a terceira e a quarta categoria não há grande variação da fração de cura.

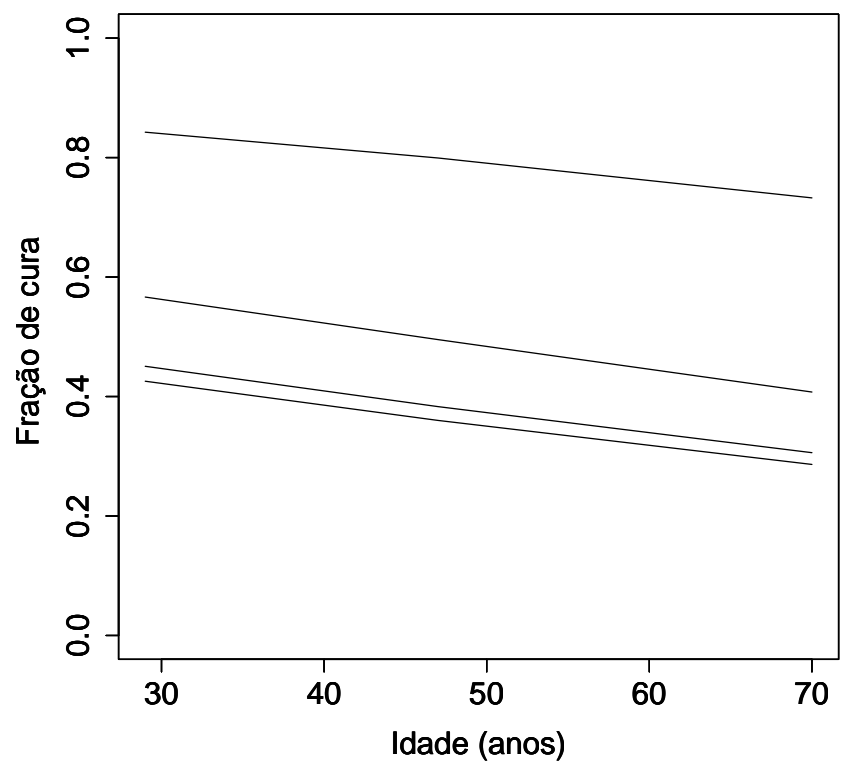

FIGURA 3.4: Proporção de curados por categoria nódulo (1 a 4, de cima para baixo). 


\subsection{Conclusões}

Neste capítulo realizamos um estudo de simulação com o objetivo de avaliar a eficiência da divergência de Kullback-Leibler em detectar observações influentes na presença de proporção de cura. Consideramos um conjunto de dados de melanoma e realizamos uma análise de diagnóstico e de sensibilidade de possíveis observações influentes com o objetivo de avaliar se tais observações influenciam na estimativa dos parâmetros do modelo. Diante de tal análise propomos um modelo final, em que optamos por excluir a covariável $x_{3}$ a qual foi não significativa. Pela Tabela 3.7 temos que as covariáveis idade e categoria do nódulo são significativas, ou seja, há uma relação entre a idade do paciente e a classificação quanto ao tumor, percebemos que a fração de cura diminui em indíviduos mais velhos e com maior categoria de nódulo. 


\section{Capítulo 4}

\section{Um modelo destrutivo com fração de}

\section{cura}

Recentemente, Rodrigues et al. (2010b) propuseram o modelo destrutivo com fração de cura, considerando a distribuição Poisson ponderada, para modelar o número inicial de causas ou riscos relacionadas à ocorrência de um particular evento de interesse. Essa proposta inclui como caso particular os modelos de sobrevivência com fração de cura proposta por Yakovlev \& Tsodikov (1996) (veja também Chen et al., 1999) e permite estimar a proporção de causas latentes que não foram removidos pelo tratamento inicial. Seguindo Rodrigues et al. (2010b), propomos um modelo destrutivo com fração de cura, assumindo que o número inicial de causas (ou riscos) latentes relacionadas à ocorrência de um particular evento de interesse é modelado pela distribuição binomial negativa apresentada no Capítulo 3. Vale ressaltar que nos modelos destrutivos temos a inclusão de um parâmetro $(p)$, que permite estimar a probabilidade do número de causas que não foram eliminadas por um tratamento inicial, por exemplo em um estudo de câncer, $p$ é responsável por avaliar o número de células que não foram eliminadas ou destruídas. Os modelos destrutivos podem ser adequados para modelar qualquer tipo de dados de sobrevivência que apresente uma fração de sobreviventes nos mais variados contextos. 


\subsection{Formulação do modelo}

Suponha que para um indivíduo da população, seja $M$ uma variável aleatória discreta que representa o número de causas que competem para produzir um evento de interesse e suponha que $M$ segue uma distribuição binomial negativa, com a função de probabilidade dada em (3.2). Suponha que dado $M=m$, sejam $W_{i}, i=1,2, \ldots, m$, variáveis aleatórias independentes de $M$, com distribuição de Bernoulli com probabilidade de sucesso $p$ indicando a presença da $i$-ésima causa competitiva. Seja a variável $N$, que representa o número total de causas competindo dentre as $M$ causas competitivas iniciais que não foram destruídas, definida como

$$
N= \begin{cases}W_{1}+W_{2}+\cdots+W_{M}, & \text { se } M>0 \\ 0, & \text { se } M=0 .\end{cases}
$$

Por danificação ou destruição, queremos dizer que $N \leq M$. O tempo (aleatório) para a $i$-ésima causa produzir o evento de interesse é denotado por $R_{i}, i=1,2, \ldots$ Assumimos que condicionado a $N$ as variáveis $R_{i}$ são i.i.d. com função distribuição acumulada $F(t)$ e função de sobrevivência $S(t)=1-F(t)$. Também assumimos que $R_{1}, R_{2}, \ldots$ são independentes de $N$. O número total de causas competindo $N$ e o tempo $R_{i}$ são não observadas e podem ser interpretadas como variáveis latentes na formulação do modelo. Então, o tempo observado para a ocorrência do evento de interesse é definida por

$$
T=\min \left\{R_{1}, R_{2}, \ldots, R_{N}\right\}
$$

com $P(T=\infty \mid N=0)=1$, o que leva a uma proporção de indivíduos "curados". As distribuições exponencial, Weibull e exponencial por partes podem ser usadas para modelar as variáveis $R_{i}$ (Ibrahim et al., 2001), como ao longo desta dissertação neste capítulo também consideramos o modelo exponencial por partes para modelar as variáveis $R_{i}$. Como observado no início do Capítulo 3, Tsodikov et al. (2003) e Rodrigues et al. 
(2008) demonstram que a função de sobrevivência populacional é dada por

$$
S_{p o p}(t)=P(T \geq t)=\phi(S(t))
$$

sendo $\phi(\cdot)$ é a função geradora de probabilidade para o número de causas competitivas $N$. Podemos mostrar que a função geradora de probabilidades para $N$ é dada por $\phi(s)=$ $[1+\alpha \theta p(1-s)]^{-1 / \alpha}$, assim a função de sobrevivência imprópria é

$$
S_{\text {pop }}(t)=\phi(S(t))=\{1+\alpha \theta p F(t)\}^{-1 / \alpha},
$$

se $p=1, S_{p o p}(t)$ em (4.3) se reduz ao modelo (3.5).

A proporção de curados é determinada por $p_{0}=\lim _{t \rightarrow \infty} S_{\mathrm{pop}}(t)=[1+\alpha \theta p]^{\frac{-1}{\alpha}} \cdot$ E a correspondente função densidade de (4.3) é expressa por

$$
\begin{aligned}
f_{\mathrm{pop}}(t) & =-\frac{d}{d t}\left(S_{p o p}(t)\right) \\
& =\theta p f(t)\{1+\alpha \theta p F(t)\}^{-(1+1 / \alpha)}
\end{aligned}
$$

com $f(t)=\frac{d}{d t}(F(t))$. Observe que $f_{\text {pop }}(t)$ não é uma função de densidade própria, já que $S_{\text {pop }}(t)$ é uma função de sobrevivência imprópria. A correspondente função de taxa de falha é dada por

$$
h_{\mathrm{pop}}(y)=\frac{\theta p f(t)}{1+\theta \alpha p F(t)}
$$

\subsection{Inferência bayesiana}

Como já dissemos anteriormente, em análise de sobrevivência é comum a existência de dados que não sejam completamente observados e estejam sujeitos a censura à direita. Seja $C_{i}$ o tempo de censura do $i$-ésimo indivíduo. Em uma amostra de tamanho $n$, observamos $Y_{i}=\min \left\{T_{i}, C_{i}\right\}$ e $\delta_{i}=\mathrm{I}\left(T_{i} \leq C_{i}\right)$, onde $\delta_{i}=1$ se $Y_{i}$ é o tempo de vida e $\delta_{i}=0$ se é tempo de censura, para $i=1, \ldots, n$. Seja $\gamma$ o vetor de parâmetros da distribuição dos tempos não observados, $R_{i 1}, \ldots, R_{i N_{i}}$. Note que o modelo destrutivo binomial negativo 
(Seção 4.1) é não identificável no sentido Li et al. (2001). Para contornar esse problema, propomos relacionar os parâmetros $p$ e $\theta$ do modelo as covariáveis $\boldsymbol{x}_{1}$ e $\boldsymbol{x}_{2}$, respectivamente, sem elementos em comum e $\boldsymbol{x}_{2}$ sem a coluna de uns. Adotamos as funções de ligações

$$
\log \left(\frac{p_{i}}{1-p_{i}}\right)=\boldsymbol{x}_{1 i}^{\top} \boldsymbol{\beta}_{1} \quad \text { e } \quad \log \left(\theta_{i}\right)=\boldsymbol{x}_{2 i}^{\top} \boldsymbol{\beta}_{2}
$$

$i=1, \ldots, n \operatorname{com} \boldsymbol{\beta}_{1}, \boldsymbol{\beta}_{2}$ denotando os vetores com $k_{1}$ e $k_{2}$ coeficientes.

A função de verossimilhança utilizada para fazermos inferências acerca dos parâmetros do modelo é expressa por

$$
L(\boldsymbol{\vartheta} ; \mathcal{D}) \propto \prod_{i=1}^{n} \prod_{j=1}^{J}\left\{\theta_{i} p_{i} f_{j}\left(y_{i} ; \boldsymbol{\lambda}\right)\right\}^{\delta_{i} \nu_{i j}}\left\{1+\alpha \theta_{i} p_{i} F_{j}\left(y_{i} ; \boldsymbol{\lambda}\right)\right\}^{-\nu_{i j}\left(\delta_{i}+1 / \alpha\right)}
$$

$\operatorname{com} \boldsymbol{\vartheta}=\left(\alpha, \boldsymbol{\beta}_{1}^{\top}, \boldsymbol{\beta}_{2}^{\top}, \boldsymbol{\lambda}^{\top}\right)^{\top}, \mathcal{D}=\left(\boldsymbol{\nu}, \boldsymbol{y}, \boldsymbol{\delta}, \boldsymbol{x}_{\mathbf{1}}, \boldsymbol{x}_{\mathbf{2}}\right), \boldsymbol{\nu}=\left(\nu_{11}, \ldots, \nu_{m J}\right) \operatorname{com} \nu_{i j}=1 \mathrm{se}$ $s_{j-1}<y_{i} \leq s_{j}$ e $\nu_{i j}=0$ caso contrário, $j=1, \ldots, J$ e $i=1, \ldots, m, \boldsymbol{y}=\left(y_{1}, \ldots, y_{n}\right)^{\top}$, $\boldsymbol{\delta}=\left(\delta_{1}, \ldots, \delta_{n}\right)^{\top}, \boldsymbol{x}_{\mathbf{1}}=\left(x_{11}, \ldots, x_{1 n}\right)^{\top}, \boldsymbol{x}_{\mathbf{2}}=\left(x_{21}, \ldots, x_{2 n}\right)^{\top}$. Enquanto que $f_{j}\left(y_{i} ; \boldsymbol{\lambda}\right)$ foi exposto na Equação $(3.7)$ e $F_{j}\left(y_{i} ; \boldsymbol{\lambda}\right)$ na Equação (3.8).

Como já visto na Seção (3.2) aqui também assumimos priori independentes para os parâmetros do modelo. Assim a densidade a priori conjunta é expressa por

$$
\pi\left(\alpha, \boldsymbol{\beta}_{1}, \boldsymbol{\beta}_{2}, \boldsymbol{\lambda}\right)=\pi(\alpha) \pi\left(\boldsymbol{\beta}_{1}\right) \pi\left(\boldsymbol{\beta}_{2}\right) \pi(\boldsymbol{\lambda})
$$

em que o parâmetro $\alpha$ tem distribuição $G(a, b), \boldsymbol{\lambda}_{i} \sim \pi\left(\boldsymbol{\lambda}_{i}\right), i=1, \ldots, J, \boldsymbol{\beta}_{\mathbf{1}} \sim N\left(0, \sigma_{1}^{2}\right)$ e $\boldsymbol{\beta}_{\mathbf{2}} \sim N\left(0, \sigma_{2}^{2}\right)$. Combinando essa densidade a priori com a verossimilhança (4.7), a densidade a posteriori conjunta é dada por

$$
\pi\left(\alpha, \boldsymbol{\beta}_{1}, \boldsymbol{\beta}_{2}, \boldsymbol{\lambda} \mid \mathcal{D}\right)=L(\boldsymbol{\gamma} ; \mathcal{D}) \pi(\alpha) \pi\left(\boldsymbol{\beta}_{1}\right) \pi\left(\boldsymbol{\beta}_{2}\right) \pi(\boldsymbol{\lambda})
$$

Como a densidade a posteriori conjunta não é uma densidade padrão usamos métodos de Monte Carlo via cadeias de Markov (MCMC), tais como o amostrador de Gibbs e Metropolis-Hasting. As densidades a posteriori condicionais para o amostrador de 
Gibbs são expressas por

$$
\begin{aligned}
& \pi\left(\boldsymbol{\beta}_{1} \mid \boldsymbol{\beta}_{2}, \alpha, \boldsymbol{\lambda}, \mathcal{D}\right) \propto L(\boldsymbol{\gamma} ; \mathcal{D}) \pi\left(\boldsymbol{\beta}_{1}\right) \\
& \pi\left(\boldsymbol{\beta}_{2} \mid \boldsymbol{\beta}_{1}, \alpha, \boldsymbol{\lambda}, \mathcal{D}\right) \propto L(\boldsymbol{\gamma} ; \mathcal{D}) \pi\left(\boldsymbol{\beta}_{2}\right) \\
& \pi\left(\boldsymbol{\lambda} \mid \boldsymbol{\beta}_{1}, \boldsymbol{\beta}_{2}, \alpha, \mathcal{D}\right) \propto L(\boldsymbol{\gamma} ; \mathcal{D}) \pi(\boldsymbol{\lambda}) \\
& \pi\left(\alpha \mid \boldsymbol{\beta}_{1}, \boldsymbol{\beta}_{2}, \boldsymbol{\lambda}, \mathcal{D}\right) \propto L(\boldsymbol{\gamma} ; \mathcal{D}) \pi(\alpha)
\end{aligned}
$$

Como as densidades a posteriori condicionais não possuem forma fechada usaremos o algoritmo de Metropolis-Hastings dentro do ciclo do algoritmo de Gibbs (Gilks et al., 1996) para gerar amostras dos parâmetros envolvidos no modelo.

\subsection{Aplicação}

Analisamos aqui o mesmo conjunto de dados descritos no Capítulo 3. Ressaltando que consideramos as covariáveis categoria do nódulo $\left(x_{1}\right)(1, n=111 ; 2, n=137 ; 3, n=87$; 4, $n=82$ ), idade $\left(x_{2}\right)$ (em anos; média $=48,0$ e desvio padrão $=13,1$ ) e espessura do tumor $\left(x_{3}\right)$ (em mm; média $=3,9$ e desvio padrão $\left.=3,2\right)$. A categoria do nódulo 1-4, respectivamente, é codificada a partir do número de gânglios envolvidos na doença $(0,1$, 2-3, e $\geq 4$ ). Para fins ilustrativos, ligamos os parâmetros $p$ em (4.6) a categoria do nódulo $\left(x_{1}\right)$ e espessura do tumor $\left(x_{3}\right)$ enquanto $\theta$ em (4.6) a idade $\left(x_{2}\right)$.

Obtemos as estimativas bayesianas considerando as seguintes distribuições a priori: para cada componente de $\boldsymbol{\beta}$, assumimos uma distribuição a priori normal com média 0 e variância $10^{3}$, para $\lambda_{j}$ assumimos uma distribuição a priori gama, ou seja, $\lambda_{j} \sim G(a, b)$ com hiperparâmetros $a=1$ e $b=0,01, j=1, \ldots, J$ e para o parâmetro $\alpha$ consideramos uma distribuição a priori informativa, $\alpha \sim G(1 ; 1)$ com a finalidade de garantir que a distribuição a posteriori seja própria (Chen et al., 1999). Geramos amostras de Gibbs através do algoritmo de Gibbs com Metropolis-Hasting da seguinte forma: geramos duas cadeias paralelas cada uma com 30.000 iterações com um burn in de 5.000 e saltos de tamanho 10, 
resultando uma amostra de Gibbs de tamanho 5.000. Para monitorar a convergência do amostrador de Gibbs utilizamos a aproximação desenvolvida por Gelman e Rubin(1992). Afim de avaliar a robustez do modelo relacionado às escolhas dos hiperparâmetros das priori, um estudo de sensibilidade foi realizado, no qual constatamos que as estimativas dos parâmetros a posteriori não apresentaram diferenças significativas. Utilizamos os critérios de seleção $B$ e $D I C$ (discutidos na Seção 1.1) para avaliar a melhor partição do eixo dos tempos. Na Tabela 4.1 apresentamos os valores para os critérios de seleção $B$ e $D I C$ para os modelos ajustados. Ambos critérios indicam $J=5$ como melhor partição do eixo dos tempos.

TABELA 4.1: Critérios de seleção para o modelo destrutivo.

\begin{tabular}{ccc}
\hline Modelo & \multicolumn{2}{c}{ Critério } \\
J & $B$ & $D I C$ \\
\hline 1 & $-536,5428$ & 1068 \\
2 & $-530,9640$ & 1061 \\
3 & $-526,5614$ & 1051 \\
4 & $-524,7432$ & 1049 \\
5 & $-516,1186$ & 1032 \\
6 & $-521,9981$ & 1044 \\
\hline
\end{tabular}

Na tabela 4.2 apresentamos os resumos a posteriori dos parâmetros do modelo destrutivo para a melhor partição. Para todos os parâmetros observamos valores de $\hat{R}$ próximos a 1, indicando que as iterações foram suficientes para se chegar a convergência. A " highest posterior density" (HPD)(Chen \& Shao, 1999) com 90\% de credibilidade indica que todas as covariáveis são significativas.

TABELA 4.2: Estimativas a posteriori para o modelo MEP destrutivo.

\begin{tabular}{ccccc}
\hline Parâmetro & Média & Desvio Padrão & $H P D(90 \%)$ & $\hat{R}$ \\
\hline$\alpha$ & 1,5381 & 0,5552 & $(0,6215 ; 2,4064)$ & 1,0664 \\
$\beta_{1_{0}}$ (intercepto) & $-4,9180$ & 1,6616 & $(-7,8063 ;-2,3406)$ & 1,0314 \\
$\beta_{1_{1}}$ (cat. nódulo) & 1,9339 & 0,7081 & $(0,8302 ; 3,0156)$ & 1,0464 \\
$\beta_{1_{3}}$ (espessura) & 0,3014 & 0,1685 & $(0,0325 ; 0,5681)$ & 1,0119 \\
$\beta_{2}$ (idade) & 0,0208 & 0,0067 & $(0,0097 ; 0,0317)$ & 1,0437 \\
$\lambda_{1}$ & 0,0767 & 0,0237 & $(0,0369 ; 0,1129)$ & 1,0721 \\
$\lambda_{2}$ & 0,2001 & 0,0541 & $(0,1132 ; 0,2680)$ & 1,0824 \\
$\lambda_{3}$ & 0,4922 & 0,1281 & $(0,2749 ; 0,6837)$ & 1,0575 \\
$\lambda_{4}$ & 0,4226 & 0,1088 & $(0,2421 ; 0,5919)$ & 1,0326 \\
$\lambda_{5}$ & 0,4328 & 0,1401 & $(0,2251 ; 0,6764)$ & 1,0414 \\
\hline
\end{tabular}


De acordo com as estimativas obtidas na Tabela 4.2, podemos notar que o sinal positivo do parâmetro $\widehat{\beta}_{2_{2}}$ indica que o número de causas competitivas aumentam ao passo que o a fração de cura diminui. Já para os parâmetros $\widehat{\beta}_{1_{1}}>0$ e $\widehat{\beta}_{1_{3}}>0$ temos que maiores valores para categoria do nódulo implica em estimativas menores para a fração de cura em ambos os casos.

Com as amostras de Gibbs foram estimadas as medidas de divergência de K-L para cada uma das 417 observações do conjunto de dados, esses resultados são graficados na Figura 4.1, em que destacamos as observações 23, 109, 176, 199, 356 e 376 por apresentar maiores valores quando comparados com as demais observações. Para verificar se essas observações são influentes procedemos como nos capítulos anteriores estimando a calibração da medida de divergência K-L, essas estimativas são apresentados na Tabela 4.3 conjuntamente com as respectivas estimativas da divergência $\mathrm{K}-\mathrm{L}$, realizamos uma análise de sensibilidade para avaliar se estas observações alteram as estimativas dos parâmetros do modelo, para tanto escolhemos as três observações com maiores valores de calibração.

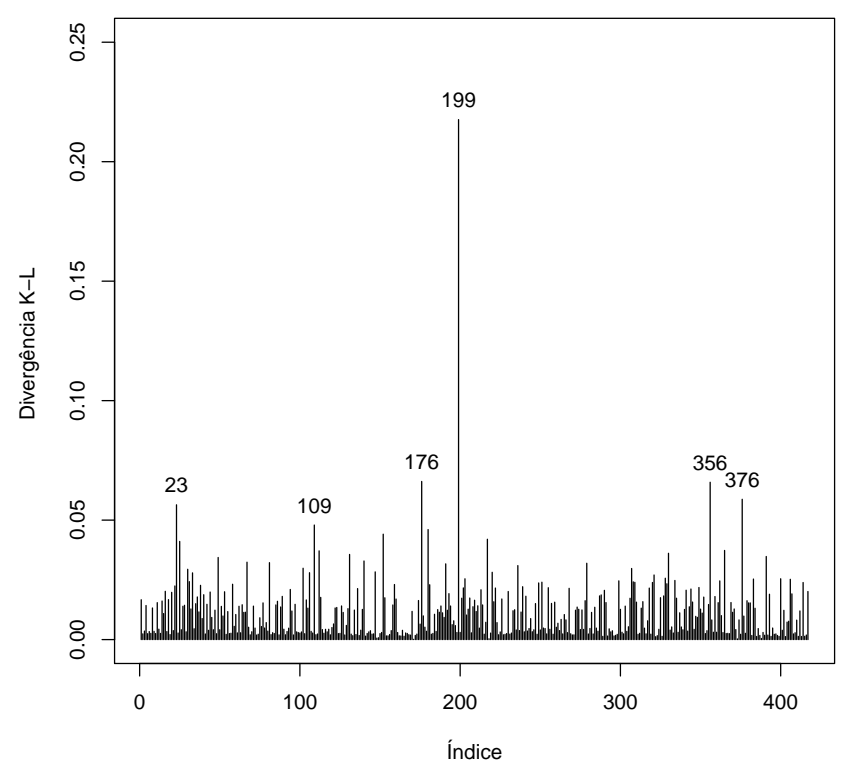

FIGURA 4.1: Gráfico de índices de $K\left(P, P_{-i}\right)$ para dados de melanoma considerando o modelo destrutivo. 
TABELA 4.3: Identificação dos casos influentes para dados de melanoma.

\begin{tabular}{ccc}
\hline Identificação do caso & \multicolumn{2}{c}{ Influência caso a caso } \\
\hline Paciente & $K\left(P, P_{-i}\right)$ & Calibração \\
\hline 23 & 0,0564 & 0,6633 \\
109 & 0,0479 & 0,6511 \\
176 & 0,0661 & 0,6760 \\
199 & 0,2176 & 0,7970 \\
356 & 0,0658 & 0,6756 \\
376 & 0,0587 & 0,6664 \\
\hline
\end{tabular}

Na Tabela 4.4 apresentamos as novas estimativas dos parâmetros após a exclusão das observações que apresentaram o maior valor de calibração, excluímos uma a uma ou todas de uma vez, representado pelo conjunto $I=\{176 ; 199 ; 356\}$, também calculamos as variações relativas $(V R)$ (em porcentagem), as quais são obtidas por $V R_{\gamma_{d}}=\mid\left(\hat{\gamma}_{d}-\right.$ $\left.\hat{\gamma}_{d(I)}\right) / \hat{\gamma}_{d} \mid \times 100 \%$, em que $\hat{\gamma}_{d(I)}$ denota a média a posteriori de $\gamma_{d}$, com $d=1, \ldots, 10$ após o conjunto $I$ de observações ter sido removido. A densidade a posteriori máxima (HPD) com $90 \%$ de credibilidade para cada nova estimativa é apresentada entre parênteses na Tabela 4.4. 
TABELA 4.4: Estimativas, $V R$ (em \%) e a correspondente HPD (90\%) ajustados para o conjunto de dados de melanoma.

\begin{tabular}{|c|c|c|c|c|}
\hline \multirow[t]{2}{*}{ Parâmetros } & \multicolumn{4}{|c|}{ Observações descartadas } \\
\hline & 176 & 199 & 356 & $I$ \\
\hline \multirow{3}{*}{$\alpha$} & 1,3205 & 1,3976 & 1,3945 & 1,6302 \\
\hline & 14,1 & 9,1 & 9,3 & 5,9 \\
\hline & $(0,3218 ; 2,1390)$ & $(0,5879 ; 2,2163)$ & $(0,5447 ; 2,2604)$ & $(0,4138 ; 2,6432)$ \\
\hline \multirow{3}{*}{$\beta_{1_{0}}($ intercepto $)$} & $-4,0530$ & $-5,6092$ & $-5,0643$ & $-6,2116$ \\
\hline & 17,5 & 14,0 & 2,9 & 26,3 \\
\hline & $(-6,7572 ;-0,5416)$ & $(-8,4381 ;-0,8271)$ & $(-7,7219 ;-0,4346)$ & $(-9,4406 ;-0,7053)$ \\
\hline \multirow{3}{*}{$\beta_{1_{1}}$ (cat. nódulo) } & 1,6533 & 2,2807 & 2,0413 & 2,5115 \\
\hline & 30,0 & 17,9 & 5,5 & 29,8 \\
\hline & $(0,3196 ; 2,7913)$ & $(0,6146 ; 3,6301)$ & $(0,4185 ; 3,2709)$ & $(0,4506 ; 4,0103)$ \\
\hline \multirow{3}{*}{$\beta_{1_{3}}($ espessura $)$} & 0,2372 & 0,3841 & 0,3234 & 0,4159 \\
\hline & 21,3 & 27,4 & 7,2 & 37,9 \\
\hline & $(-0,0785 ; 0,4990)$ & $(-0,0401 ; 0,7055)$ & $(-0,0474 ; 0,6140)$ & $(-0,0380 ; 0,7578)$ \\
\hline \multirow{3}{*}{$\beta_{2_{2}}($ idade $)$} & 0,0170 & 0,0182 & 0,0185 & 0,0202 \\
\hline & 18,2 & 12,5 & 11,0 & 2,8 \\
\hline & $(0,0048 ; 0,0276)$ & $(0,0081 ; 0,0279)$ & $(0,0071 ; 0,0297)$ & $(0,0081 ; 0,0330)$ \\
\hline \multirow[t]{2}{*}{$\lambda_{1}$} & 0,0979 & 0,0852 & 0,0878 & 0,0787 \\
\hline & 27,6 & 11,0 & 14,4 & 2,6 \\
\hline \multirow[t]{2}{*}{$\lambda_{2}$} & 0,2218 & 0,2013 & 0,1995 & 0,2042 \\
\hline & 10,8 & 0,5 & 0,2 & 2,0 \\
\hline \multirow[t]{2}{*}{$\lambda_{3}$} & 0,5605 & 0,4906 & 0,5218 & 0,5304 \\
\hline & 13,8 & 0,3 & 6,0 & 7,7 \\
\hline \multirow[t]{2}{*}{$\lambda_{4}$} & 0,4728 & 0,4186 & 0,4413 & 0,4336 \\
\hline & 11,8 & 0,9 & 4,4 & 2,6 \\
\hline \multirow[t]{2}{*}{$\lambda_{5}$} & 0,5225 & 0,4491 & 0,4640 & 0,5024 \\
\hline & 20,7 & 3,7 & 7,2 & 16,0 \\
\hline Critério $B$ & $-516,8554$ & $-516,1045$ & $-516,4568$ & $-506,8090$ \\
\hline
\end{tabular}

Observando a Tabela 4.4 percebemos maiores valores de $V R$ para o parâmetro $\widehat{\beta}_{1_{3}}$ quando há exclusão da observação influente 199 e do conjunto $I$, o que também ocorre com o parâmetro $\widehat{\beta}_{1_{1}}$ quando excluímos a observação 176 , com relação aos parâmetros $\widehat{\lambda}$ temos as maiores variações relativas $(V R)$ quando excluímos a observação 176 . O parâmetro $\widehat{\beta}_{1_{3}}$ apresenta sensibilidade em relação as observações influentes, pois quando excluímos qualquer um dos casos ou todos, há alteração na significância do parâmetro, resumidamente, $\widehat{\beta}_{1_{3}}$ passa a ser não significativo no modelo. Os demais parâmetros não apresentam mudanças significativas.

Sugerimos como alternativa a um modelo final excluir o conjunto de observações $I$ de acordo com o critério de seleção de modelos $(B)$ (Tabela 4.4) e a covariável $x_{3}$, uma vez que ela passa a ser não significativa após a exclusão de observações influentes. Um resumo a posteriori dos parâmetros do modelo final é apresentado na Tabela 4.5. 
TABELA 4.5: Resumo dos parâmetros a posteriori para o modelo final.

\begin{tabular}{cccc}
\hline Parâmetro & Média & Desvio Padrão & HPD $(90 \%)$ \\
\hline$\alpha$ & 1,5449 & 0,6448 & $(0,4987 ; 2,5224)$ \\
$\beta_{1_{0}}$ (intercepto) & $-2,6957$ & 0,5930 & $(-3,6777 ;-1,7810)$ \\
$\beta_{1_{1}}$ (cat. nódulo) & 1,1031 & 0,3710 & $(0,5724 ; 1,6613)$ \\
$\beta_{2_{2}}$ (idade) & 0,0233 & 0,0074 & $(0,0111 ; 0,0353)$ \\
$\lambda_{1}$ & 0,0854 & 0,0280 & $(0,0391 ; 0,1286)$ \\
$\lambda_{2}$ & 0,2197 & 0,0611 & $(0,1224 ; 0,3210)$ \\
$\lambda_{3}$ & 0,5650 & 0,1440 & $(0,3235 ; 0,8024)$ \\
$\lambda_{4}$ & 0,4532 & 0,1149 & $(0,2631 ; 0,6318)$ \\
$\lambda_{5}$ & 0,5362 & 0,1767 & $(0,2624 ; 0,8163)$ \\
\hline
\end{tabular}

Obtivemos os valores dos critérios de seleção, $B=-509,6407$ e $D I C=1019$, ao compará-los aos valores da Tabela 4.1 temos que ao excluir as observações $I$ e a covariável $x_{3}$, obtemos um modelo com melhor ajuste.

Assim como no Capítulo 3 as maiores alterações nos resumos a posteriori dos parâmetros do modelo estão relacionados aos coeficientes $\boldsymbol{\beta}$.

Na Tabela 4.6 apresentamos as estimativas baseadas em 5000 amostras da distribuição a posteriori dos parâmetros para o modelo final destrutivo, as quais revelam que quanto maior a categoria do nódulo maior a probabilidade de células não destruídas. A probabilidade da presença de causas competindo estratificada por categoria do nódulo em (4.6) é dada por $p_{i}=\exp \left(\beta_{1_{0}}+x_{1_{i}} \beta_{1_{1}}\right) /\left\{1+\exp \left(\beta_{1_{0}}+x_{1_{i}} \beta_{1_{1}}\right)\right\}$. Notamos ainda ao nível de $5 \%$ uma diferença significativa entre a primeira e a última categoria do nódulo.

TABELA 4.6: Resumo a posteriori para a probabilidade da presença de causas competindo $(p)$ estratificada por categoria do nódulo.

\begin{tabular}{ccccc}
\hline Probabilidade & & & \multicolumn{2}{c}{ Percentil } \\
& Média & Desvio padrão & $2,5 \%$ & $97,5 \%$ \\
\hline$p_{1}$ & 0,1776 & 0,0630 & 0,0783 & 0,3241 \\
$p_{2}$ & 0,3861 & 0,1236 & 0,1877 & 0,6745 \\
$p_{3}$ & 0,6269 & 0,1559 & 0,3281 & 0,9292 \\
$p_{4}$ & 0,8016 & 0,1338 & 0,4871 & 0,9888 \\
\hline
\end{tabular}


TABELA 4.7: Resumo a posteriori para a fração de cura $\left(p_{0}\right)$ estratificada por categoria do nódulo e por idade.

\begin{tabular}{cccccc}
\hline \multirow{2}{*}{ Idade } & Fração de Cura & Média & Desvio Padrão & $2,50 \%$ & $97,50 \%$ \\
\hline \multirow{2}{*}{29} & $p_{0_{1}}$ & 0,7640 & 0,0420 & 0,6771 & 0,8404 \\
& $p_{0_{2}}$ & 0,6156 & 0,0427 & 0,5275 & 0,6991 \\
& $p_{0_{3}}$ & 0,5085 & 0,0378 & 0,4361 & 0,5860 \\
47 & $p_{0_{4}}$ & 0,4513 & 0,0357 & 0,3823 & 0,5203 \\
& $p_{0_{1}}$ & 0,6894 & 0,0439 & 0,6019 & 0,7711 \\
& $p_{0_{2}}$ & 0,5280 & 0,0374 & 0,4482 & 0,5967 \\
70 & $p_{0_{3}}$ & 0,4223 & 0,0366 & 0,3491 & 0,4906 \\
& $p_{0_{4}}$ & 0,3691 & 0,0417 & 0,2867 & 0,4514 \\
& $p_{0_{1}}$ & 0,5814 & 0,0570 & 0,4694 & 0,6923 \\
& $p_{0_{2}}$ & 0,4182 & 0,0440 & 0,3296 & 0,5033 \\
& $p_{0_{3}}$ & 0,3234 & 0,0457 & 0,2350 & 0,4131 \\
& $p_{0_{4}}$ & 0,2788 & 0,0522 & 0,1755 & 0,3838 \\
\hline
\end{tabular}

A Figura 4.2 ilustra os resultados apresentados na Tabela 4.7, ou seja, a proporção de cura por categoria do nódulo de acordo com as idades de 29, 47, e 70 anos, que correspondem aos quantis como descrito no Capítulo 3. É fácil notar que com o aumento da idade e quanto maior a categoria do nódulo, menor a fração de cura. Há uma diferença significativa na proporção de curados entre a primeira categoria de nódulo e a última. Já entre a terceira e a quarta categoria não há grande variação da fração de cura.

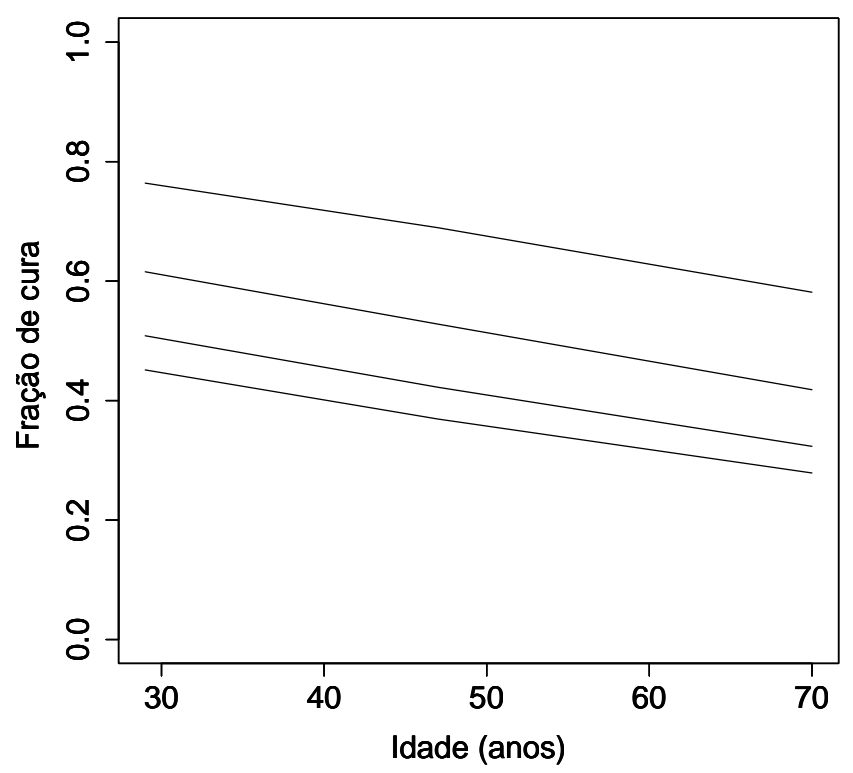

FIGURA 4.2: Proporção de curados por categoria nódulo (1 a 4, de cima para baixo). 


\subsection{Conclusões}

Com relação ao exposto neste capítulo, de maneira geral, notamos que modelo proposto permite estimar a probabilidade da presença de causas competitivas após um tratamento inicial. Observamos que a probabilidade de sobrevivência diminui com a idade dos pacientes e que a fração de cura é mais baixa para pacientes com maior categoria de nódulo. Na aplicação do conjunto de dados referentes ao estudo de melanoma, observamos que o modelo destrutivo com fração de cura proporciona um melhor ajuste se comparado com o modelo abordado no Capítulo 3, o que podemos notar pelo critério de seleção de modelos. 


\section{Capítulo 5}

\section{Considerações Finais e Propostas}

\section{Futuras}

Com os estudos desenvolvidos ao longo deste texto, observamos que o uso do modelo exponencial por partes é adequado para estimar os tempos de vida, além de ser um modelo que apresenta grande flexibilidade ao acomodar funções de taxa de falha com diversas formas. Determinamos a melhor partição do eixo dos tempos de acordo com os principais critérios de seleção de modelos. Sendo esta uma questão fundamental para o processo de inferência.

Podemos observar que a divergência de Kullback-Leibler detecta os pontos influentes no modelo, o que pudemos comprovar por meio dos estudos de simulação e dos dados reais.

Com relação aos modelos destrutivos observamos que eles proporcionam um melhor ajuste (para o conjunto de dados considerado nesta dissertação), se comparados aos modelos com fração de cura, o que pudemos observar pelos critérios de seleção de modelos. Foi possível ainda, calcular a proporção de curados e estimar a probabilidade de causas competindo após um tratamento inicial, ou seja células que ainda não foram destruídas pelo tratamento.

Verificamos que o uso de métodos Bayesianos com técnicas de simulação de Monte 
Carlo em Cadeias de Markov (MCMC), são apropriadas para a obtenção de sumários a posteriori de interesse.

A implementação computacional foi desenvolvida nos sistemas OpenBUGS (Spiegelhalter et al., 2007) e R (R Development Core Team, 2010), para detalhes do programa pode ser consultado aos autores do trabalho

Como propostas futuras podemos realizar um estudo de diagnóstico embasado na abordagem clássica utilizando a técnica de influência local proposta por Cook (1986). Considerar os macanismos de ativação com base em fatores latentes encontrado em Cooner et al. (2007) utilizando tanto aboradagem clássica como bayesiana. 


\section{Referências}

Aitkin, M., Laird, N. \& Francis, B. (1983). Covariance analysis of censured survival data. Journal of the American Statistical Association, 78, 264-292.

Barbosa, E., Colosimo, E. \& Louzada-Neto, F. (1996). Accelerated life tests analyzed by a piecewise exponential distribution via generalized linear models. IEEE Transactions on Reliability, 45, 619-623.

Barlow, R. \& Campo, R. (1975). Total Time on Test Processes and Applications to Failure Data Analysis. California University Berkeley Operations Research Center.

Berkson, J. \& Gage, R. P. (1952). Survival cure for cancer patients following treatment. Journal of the American Statistical Association, 47, 501-515.

Bernardo, J. \& Smith, A. (2000). Bayesian Theory. John Wiley \& Sons.

Boag, J. (1949). Maximum likelihood estimates of the proportion of patients cured by cancer therapy. Journal of the Royal Statistical Society. Series B, 11, 15-53.

Breslow, N. (1974). Covariance analysis of censured survival data. Biometrics, 30, 34-41.

Brookmeyer, R. \& Goedert, J. J. (1989). Censoring in an epidemic with an application to hemophilia-associated aids. Biometrics, 45, 325-335.

Cancho, V., Ortega, E. \& Bolfarine, H. (2009). The Log-exponentiated-Weibull Regression Models with Cure Rate: Local Influence and Residual Analysis. Journal of Data Science, 7, 433-458.

Cancho, V., Ortega, E. \& Paula, G. (2010). On estimation and influence diagnostics for log-Birnbaum-Saunders Student-t regression models: Full Bayesian analysis. Journal of Statistical Planning and Inference, 140, 2486-2496.

Cancho, V. G., Rodrugues, J. \& de Castro, M. (2011). A flexive model for survival with a cure rate: A bayesian approach. Journal of Applied Statistics, 38, 57-70.

Carrasco, J. M. F. (2007). Modelo de Regressão Log-Weibull Modificado e a Nova Distribuição Weibull Modificada Generalizada. Dissertação de mestrado, Universidade de São Paulo - Escola Superior de Agricultura "Luiz de Queiroz", Piracicaba.

Chen, J., Ayyagari, R., Chatterjee, N., Pee, D., Schairer, C., Byrne, C., Benichou, J. \& Gail, M. H. (2008). Breast Cancer Relative Hazard Estimates From Case-Control and Cohort Designs With Missing Data on Mammographic Density. Journal of the American Statistical Association, 103, 976-988. 
Chen, M. \& Shao, Q. (1999). Monte Carlo estimation of Bayesian credible and HPD intervals. Journal of Computational and Graphical Statistics, 8, 69-92.

Chen, M.-H. \& Ibrahim, J. G. (2001). Maximum likelihood methods for cure rate models with missing covariates. Biometrics, 57, 43-52.

Chen, M. H., Ibrahim, J. G. \& Sinha, D. (1999). A new bayesian model for survival data with a surviving fraction. Journal of the American Statistical Association, 94, 909-919.

Chen, M. H., Shao, Q. M. \& Ibrahim, J. G. (2000). Monte Carlo methods in Bayesian computation. Springer-Verlag, New York.

Chen, M. H., Harrington, D. \& Ibrahim, J. (2002). Bayesian cure rate models for malignant melanoma: a case-study of Eastern Cooperative Oncology Group trial E1690. Applay Statistics, 51, 135-150.

Chi, Y. Y. \& Ibrahim, J. G. (2007). Bayesian approaches to joint longitudinal and survival models accommodating both zero and nonzero cure fractions. Statistica Sinica, 17, 445-462.

Cho, H., Ibrahim, J. G., Sinha, D. \& Shu, H. (2009). Bayesian case influence diagnostics for survival models. Biometrics, 65, 116-124.

Clark, D. \& Ryan, L. (2002). Concurrent prediction of hospital mortality and length of stay from risk factors on admission. Health Services Research, 37, 631-645.

Colosimo, E. A. \& Giolo, S. R. (2006). Análise de Sobrevivência Aplicada. Editora Edgard Blücher, Brasil.

Cook, R. (1986). Assessment of local influence. Journal of the Royal Statistical Society. Series B (Methodological), 48, 133-169.

Cook, R. \& Weisberg, S. (1982). Residuals and influence in regression. Chapman and Hall New York.

Cooner, F., Banerjee, S., Carlin, B. \& Sinha, D. (2007). Flexible cure rate modeling under latent activation schemes. Journal of the American Statistical Association, 102, 560-572.

Cox, D. (1972). Regression models and life-tables. Journal of the Royal Statistical Society. Series B (Methodological).

Cox, D. R. \& Snell, E. J. (1968). A general definition of residuals. Journal of the Royal Statistical Society, 30, 248-275.

de Castro, M., Cancho, V. \& Rodrigues, J. (2009). A Bayesian Long-term Survival Model Parametrized in the Cured Fraction. Biometrical Journal, 51, 443-455.

de Castro, M., Cancho, V. \& Rodrigues, J. (2010). A hands-on approach for fitting longterm survival models under the GAMLSS framework. Computer methods and programs in biomedicine, $\mathbf{9 7}(2), 168-177$.

Demarqui, F., Loschi, R. \& Colosimo, E. (2008). Estimating the grid of time-points for the piecewise exponential model. Lifetime Data Analysis, 14, 333-356. 
Fonseca, R. (2009). Modelos de sobrevivência com fração de cura e omissão nas covariáveis. Tese de doutorado, Universidade Federal do Rio Grande do Norte.

Friedman, M. (1982). Piecewise exponential models for survival data with covariates. The Annals of Statistics, 10, 101-113.

Gamerman, D. (1994). Bayes estimation of the piece-wise exponential distribution. IEEE Transactions on Reliability, 43, 128-131.

Gilks, W., Gilks, W., Richardson, S. \& Spiegelhalter, D. (1996). Markov chain Monte Carlo in practice. Chapman \& Hall/CRC.

Hoggart, C. J. \& Griffin, J. E. (2001). A Bayesian partition model for customer attrition. In E. I. George, editor, Bayesian Methods with Applications to Science, Policy, and Official Statistics (Selected Papers from ISBA 2000 - Creta, Greece), pages 61-70. International Society for Bayesian Analysis.

Ibrahim, J., Chen, M. \& Sinha, D. (2001). Bayesian survival analysis. Springer Verlag.

Kim, J. \& Proschan, F. (1991). Piecewise exponential estimator of the survivor function. IEEE Transactions on Reliability, 40, 134-139.

Kim, S., Chen, M. H., Dey, D. K. \& Gamerman, D. (2007). Bayesian dynamic models for survival data with a cure fraction. Lifetime Data Analysis, 13, 17-35.

Labra, F. V., Aoki, R. \& Bolfarine, H. (2005). Local influence in null intercept measurement error regression under a student-t model. Journal of Applied Statistics, $32,723-740$.

Lawless, J. \& Lawless, J. (1982). Statistical models and methods for lifetime data. Wiley New York.

Li, C., Taylor, J. \& Sy, J. (2001). Identifiability of cure models. Statistics \& Probability Letters, 54(4), 389-395.

Lopes, C. (2008). Modelos de sobrevivência com fração de cura e efeitos aleatórios. Tese de doutorado, Universidade de São Paulo.

Maller, R. A. \& Zhou, X. (1996). Survival Analysis with Long-Term Survivors. Wiley, New York.

McCulloch, R. E. (1989). Local model influence. Journal of the American Statistical Association, 84, 473-478.

McGilchrist, C. \& Aisbett, C. (1991). Regression with frailty in survival analysis. Biometrics, 47, 461-466.

Mizoi, M. \& Bolfarine, H. (2007). Cure Rate Model with Measurement Error. Communications in Statistics: Simulation and Computation, 36, 185-196.

Mizoi, M. F. (2004). Influência local em modelos de sobrevivência com fração de cura. Tese de doutorado, Instituto de Matemática e Estatística - Universidade de São Paulo, São Paulo. 
Nelson, W. (1990). Accelerated testing: statistical models, test plans and data analyses. Wiley New York.

Ortega, E., Bolfarine, H. \& Paula, G. (2003). Influence diagnostics in generalized loggamma regression models. Computational Statistics \& Data Analysis, 42, 165-186.

Ortega, E., Cancho, V. \& Paula, G. (2009). Generalized log-gamma regression models with cure fraction. Lifetime Data Analysis, 15, 79-106.

Ortega, E. M. M., Cancho, V. G. \& Bolfarine, H. (2006). Influence diagnostics in exponentiated-Weibull regression models with censored data. SORT. Statistics and Operations Research Transactions, 30, 171-192.

Paes, A. (2007). Uso de modelos com fração de cura na análise de dados de sobrevivência com omissão nas covariáveis. Tese de doutorado, Universidade de São Paulo.

Paula, G. A. (2004). Modelos de Regressão com Apoio Computacional. Instituto de Matemática e Estatística - Universidade de São Paulo, Brasil.

Peng, F. \& Dey, D. (1995). Bayesian analysis of outlier problems using divergence measures. Canadian Journal of Statistics, 23, 199-213.

Piegorsch, W. W. (1990). Maximum likelihood estimation for the negative binomial dispersion parameter. Biometrics, 46, 863-867.

R Development Core Team (2010). R: A Language and Environment for Statistical Computing. R Foundation for Statistical Computing, Vienna, Austria.

Reineke, D., Pohl, E. \& Murdock Jr, W. (1999). Maintenance-policy cost-analysis for a series system withhighly-censored data. IEEE Transactions on Reliability, 48, 413-419.

Rodrigues, J., Cancho, V. G. \& de Castro, M. (2008). Teoria Unificada de Análise de Sobrevivência. ABE, Brasil.

Rodrigues, J., Cancho, V., de Castro, M. \& Louzada-Neto, F. (2009a). On the unification of long-term survival models. Statistics and Probability Letters, 79, 753-759.

Rodrigues, J., de Castro, M., Cancho, V. \& Balakrishnan, N. (2009b). COM-Poisson cure rate survival models and an application to a cutaneous melanoma data. Journal of Statistical Planning and Inference, 139, 3605-3611.

Rodrigues, J., Cancho, V., Castro, M. \& Balakrishnan, N. (2010a). A Bayesian destructive weighted Poisson cure rate model and an application to a cutaneous melanoma data. Statistical Methods in Medical Research.

Rodrigues, J., de Castro, M., Balakrishnan, N. \& Cancho, V. (2010b). Destructive weighted Poisson cure rate models. Lifetime Data Analysis.

Schmidli, H., Bretz, F. \& Racine-Poon, A. (2007). Bayesian predictive power for interim adaptation in seamless phase II/III trials where the endpoint is survival up to some specified timepoint. Statistics in medicine, 26, 4925-4938.

Sen, A. \& Tan, F. (2008). Cure-rate estimation under Case-1 interval censoring. Statistical Methodology, 5, 106-118. 
Spiegelhalter, D., Best, N., Carlin, B. \& van der Linde, A. (2002). Bayesian measures of model complexity and fit. Journal of the Royal Statistical Society: Series B (Statistical Methodology), 64, 583-639.

Spiegelhalter, D., Thomas, A., Best, N. \& Lunn, D. (2007). Openbugs: User manual, version 3.0. 2. MRC Biostatistics Unit, Cambridge.

Tournoud, M. \& Ecochard, R. (2007). Application of the promotion time cure model with time-changing exposure to the study of HIV/AIDS and other infectious diseases. Statistics in medicine, 26, 1008-1021.

Tsodikov, A. D., Ibrahim, J. G. \& Yakovlev, A. Y. (2003). Estimating cure rates from survival data: an alternative to two-component mixture models. Journal of the American Statistical Association, 98, 1063-1078.

Yakovlev, A. Y. \& Tsodikov, A. D. (1996). Stochastic Models of Tumor Latency and Their Biostatistical Applications. World Scientific, New Jersey.

Yamaguchi, K. (1992). Accelerated Failure-Time Regression Models with a Regression Model of Surviving Fraction: An Application to the Analysis of "Permanent Employment" in Japan. Journal of the American Statistical Association, 87, 284-292.

Yin, G. (2005). Bayesian cure rate frailty models with application to a root canal therapy study. Biometrics, 61, 552-558.

Zaider, M., Zelefsky, M. J., Hanin, L. G., Tsodikov, A. D., Yakovlev, A. Y. \& Leibel, S. A. (2001). A survival model for fractionated radiotherapy with an application to prostate cancer. Physics in Medicine and Biology, 46, 2745-2758. 